\title{
The Peculiar Globular Cluster Palomar 1 and Persistence in the SDSS-APOGEE Database
}

\author{
Farbod Jahandar ${ }^{1 \star}$, Kim A. Venn ${ }^{1}$, Matthew D. Shetrone ${ }^{2}$, Mike Irwin $^{3}$, Jo \\ Bovy $^{4,5} \uparrow$, Charli M. Sakari ${ }^{6}$, Collin L. Kielty ${ }^{1}$, Ruth A. R. Digby ${ }^{1}$ and Peter M. \\ Frinchaboy $^{7}$ \\ ${ }^{1}$ Department of Physics and Astronomy, University of Victoria, Victoria, BC, V8W 3P2, Canada \\ ${ }^{2}$ Mcdonald Observatory, University of Texas at Austin, HC75 Box 1337-MCD, Fort Davis, TX 79734, USA \\ ${ }^{3}$ Institute of Astronomy, University of Cambridge, Cambridge, CB3 OHA, UK \\ ${ }^{4}$ Department of Astronomy and Astrophysics, University of Toronto, Toronto, ON, M5S 3H4, Canada \\ ${ }^{5}$ Center for Computational Astrophysics, Flatiron Institute, 162 5th Ave, New York, NY 10010, USA \\ ${ }^{6}$ Department of Astronomy, University of Washington, Seattle, WA, 98195-1580, USA \\ ${ }^{7}$ Department of Physics and Astronomy, Texas Christian University, TCU Box 298840, Fort Worth, TX 76129, USA
}

Accepted XXX. Received YYY; in original form ZZZ

\begin{abstract}
The SDSS-III APOGEE DR12 is a unique resource to search for stars beyond the tidal radii of star clusters. We have examined the APOGEE DR12 database for new candidates of the young star cluster Palomar 1, a system with previously reported tidal tails (Niederste-Ostholt et al. 2010). The APOGEE ASPCAP database includes spectra and stellar parameters for two known members of Pal 1 (Stars I and II), however these do not agree with the stellar parameters determined from optical spectra by Sakari et al. (2011). We find that the APOGEE analysis of these two stars is strongly affected by the known persistence problem (Majewski et al. 2015; Nidever et al. 2015). By re-examining the individual visits, and removing the blue (and sometimes green) APOGEE detector spectra affected by persistence, then we find excellent agreement in a re-analysis of the combined spectra. These methods are applied to another five stars in the APOGEE field with similar radial velocities and metallicities as those of Pal 1. Only one of these new candidates, Star F, may be a member located in the tidal tail based on its heliocentric radial velocity, metallicity, and chemistry. The other four candidates are not well aligned with the tidal tails, and comparison to the Besançon model (Robin et al. 2003) suggests that they are more likely to be non-members, i.e. part of the Galactic halo. This APOGEE field could be re-examined for other new candidates if the persistence problem can be removed from the APOGEE spectral database.
\end{abstract}

Key words: stars: chemical abundances - techniques: spectroscopy - globular clusters: individual (Palomar 1) - globular clusters: tidal tail

\section{INTRODUCTION}

Palomar 1 (Pal1) is an unusual globular cluster. It is young (4-6 Gyr; Sarajedini et al. 2007) and it has a high metallicity $([\mathrm{Fe} / \mathrm{H}]=-0.6 \pm 0.1$; Sakari et al. 2011; Monaco et al. 2011); however, it is located $3.6 \mathrm{kpc}$ above the Galactic plane, and $17.2 \mathrm{kpc}$ from the Galactic Centre (Harris 1996, 2010 edition). Niederste-Ostholt et al. (2010) examined SDSS and HST photometric fields around Pal1, and detected a dis-

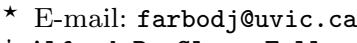

$\dagger$ Alfred P. Sloan Fellow
}

persed tidal tail extending up to $1^{\circ}(\sim 0.4 \mathrm{kpc}$, or $\sim 80$ half-light radii) from either side of the cluster centre, with roughly as many stars in the tails as in the central cluster region.

Examination of the chemical abundances of the stars in Pall can be used to study the origin of this system. If Pal1 is a globular cluster that has been shredded, then its stars should show a Na-O anti-correlation (Carretta et al. 2010). However, if Pal1 is a captured stellar group from a dwarf galaxy, then it can be expected to show lower ratios of the $\alpha$-elements (amongst other chemical signatures, e.g., see Venn et al. 2004; Tolstoy et al. 2009; Frebel \& Norris 2015). 
Sakari et al. (2011) determined the elemental abundances of five stars in Pal1 from high-resolution HDS Subaru spectroscopy. There was no evidence for a Na-O anti-correlation in the sample, and the $[\alpha / \mathrm{Fe}]$ ratios were slightly lower than Galactic field stars at the same metallicity but only with $1 \sigma$ significance. These signatures do not favour either scenario for the origin of Pal1; however, Sakari et al. (2011) also found high values of $[\mathrm{Ba} / \mathrm{Y}]$ and $[\mathrm{Eu} / \alpha]$ that indicate unique contributions of r-process elements in this system, which seem to differ from most Galactic stars.

The physical properties of Pal1 more closely resemble those of young clusters associated with the Sgr stream (i.e. Pal12 and Ter7; Sakari et al. 2011), or the intermediate-age clusters in the LMC (Sakari et al. 2017; Mucciarelli et al. 2008; Hill et al. 2000). Like Pal1, those clusters also have young ages determined from isochrone fitting (Dotter et al. 2008; Siegel et al. 2007; Salaris \& Weiss 2002) and show lower $[\alpha / \mathrm{Fe}]$ ratios for their metallicities (Sbordone et al. 2007; Cohen 2004; Bonifacio et al. 2004). Furthermore, neither Pal1, nor the other young halo clusters, show the sodium-oxygen anti-correlation that Carretta et al. (2010) have shown is typical of globular clusters in the Milky Way. Another interesting sparse and young cluster in the halo is Rup106. Like Pal1, Rup106 also has low $[\alpha / \mathrm{Fe}]$ for its metallicity and no Na-O anti-correlation (Villanova et al. 2013). Rup106 is not associated with any stellar streams, unlike the Sgr clusters. However, Rup106 also shows low $[\mathrm{La} / \mathrm{Fe}]$ and $[\mathrm{Na} / \mathrm{Fe}]$, so does not appear to be directly linked to Pal1. Pall may also be linked to the Canis Major over-density based on its chemistry, e.g., high [Ba/Fe] and [La/Fe] (Sakari et al. 2011; Martin et al. 2004; Chou et al. 2010).

If Pal1 is a tidally disrupted globular cluster, this makes it an excellent probe of the shape of the Milky Way halo. Palomar 5 (Pal5), another low-mass, low-velocity dispersion globular cluster with more spectacular tidal tails, has been used to model the Galactic potential by Bovy et al. (2016b), Ishigaki et al. (2016) (2016), Grillmair (2006), and Belokurov et al. (2007). Pal5 also shows gaps in the tidal tails that have been examined for constraints on mini-halo substructure (Bovy et al. 2016a; Carlberg et al. 2012). The tidal tails around Pal1 are much shorter. Characterizing this system further by identifying member stars in the tidal tails, or in a more extended envelope, could be used to better study the shape of the Milky Way halo and the origin and evolution of this cluster.

In this paper, we examine the SDSS-APOGEE DR 12 database, which targeted Pall as part of its globular cluster ancillary data project. Our search for new members of Pal1 required a critical and substantial re-examination of the individual visit spectra and data analysis techniques. In this paper, we present our target selection methods, and cleaning of the combined spectra to remove the persistence problem, and re-analysis of the stellar parameters using the FERRE pipeline. We compare the results with those from Sakari et al. (2011) and Niederste-Ostholt et al. (2010), as well as with the Besançon model (Robin et al. 2003).

\section{APOGEE DATA}

The Apache Point Observatory Galactic Evolution Experiment (APOGEE) is a high-resolution, high signal-to-noise

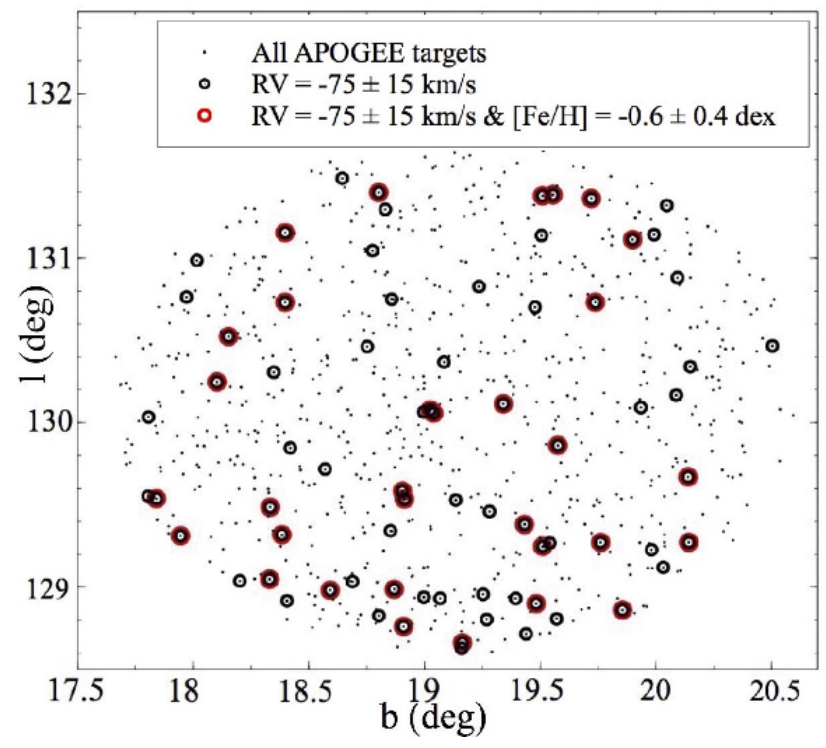

Figure 1. Position of each star from the APOGEE DR12 database in the Pall field in Galactic coordinates. Those with heliocentric radial velocities and metallicities similar to those for Pall are noted in red circles.

infrared (IR) spectroscopic survey of over 100,000 red giant stars across the full range of the Galactic bulge, bar, disk, and halo (Majewski et al. 2015). The survey was carried out at the 2.5-m Sloan Foundation Telescope in New Mexico, covering the wavelength range from 1.5 to 1.7 microns in the $\mathrm{H}$ band, with spectral resolution $\mathrm{R}=22,500$ (Gunn et al. 2006). The APOGEE Stellar Parameters and Chemical Abundances Pipeline (ASPCAP) DR12 (Pérez et al. 2016) is a data analysis pipeline that produces stellar parameters and abundances for 15 different elements (C, N, O, Na, Mg, $\mathrm{Al}, \mathrm{Si}, \mathrm{S}, \mathrm{K}, \mathrm{Ca}, \mathrm{Ti}, \mathrm{V}, \mathrm{Mn}, \mathrm{Fe}$ and $\mathrm{Ni}$ ).

APOGEE uses the same field size and target positioner as the Sloan Extension for Galactic Understanding and Exploration (SEGUE) of the Sloan Digial Sky Survey (SDSS). It uses a series of 7 squared degree tiles to sample the sky with 2" fibres that observe 300 targets simultaneously. One of these tiles was centred on Pal1 $\left(\mathrm{RA}=53.33^{\circ} \& \mathrm{Dec}=79.58^{\circ}\right.$, Harris 1996, 2010 edition) with fibers allocated to a variety of targets based on the colours of cool stars (see target selection for the APOGEE program by Zasowski et al. 2013). Foreground dwarfs are removed from our analysis, as well as objects that are unlikely to be associated with Pall based on their metallicity and radial velocity. These include objects with radial velocities outside of $-75 \pm 15 \mathrm{kms}^{-1}$ and metallicities outside of $-1.0<[\mathrm{Fe} / \mathrm{H}]<-0.2$ (i.e., $4 \sigma$ and $2 \sigma$ of the values for confirmed Pall members respectively, e.g., Rosenberg 1998, to account for errors in the APOGEE metallicities and potential kinematic effects along the tidal tails). These targets are shown in Fig. 1, where $9 \%$ of the stars in this field may be associated with Pal1. Two of these are Stars I and II examined from optical spectra by Sakari et al. (2011). To further select Pal1 members, we examine a colour-magnitude diagram (CMD) of stars in the central portion of Pal1 from HST ACS photometry (Sarajedini et al. 2007); see Fig. 2. Isochrones are generated from the Dart- 

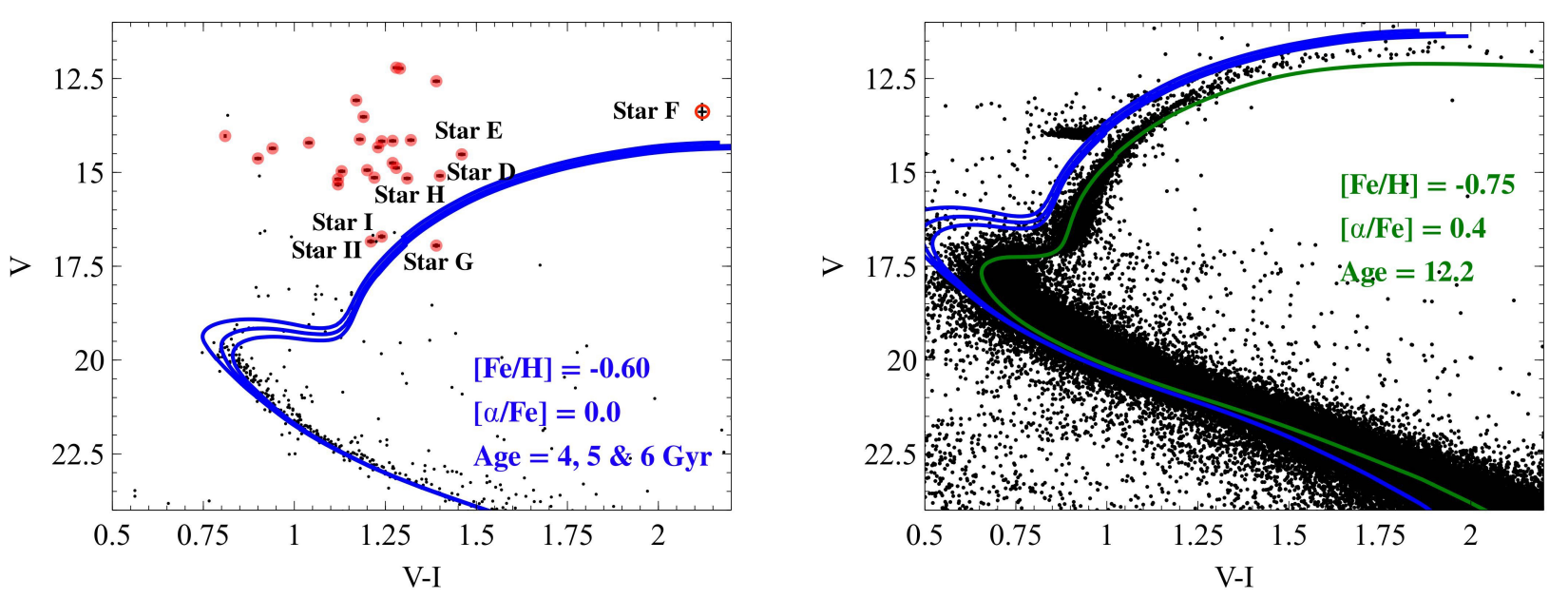

Figure 2. The left panel shows a color-magnitude diagram for Pal 1 (from Sarajedini et al. 2007) with three isochrones for ages 4,5 and 6 Gyr, from the Dartmouth Stellar Evolution Database (Dotter et al. 2008) and the right panel shows the color-magnitude diagram of 47 Tuc (from Sarajedini et al. 2007) and an isochrone for age 12.2 Gyr as a reference for the position of the red giant branch in a typical globular cluster. Note that the distance modulus and reddening of 47 Tuc is applied to the isochrones of Pal1 in the right panel in order to compare age of the clusters. All of the APOGEE stars with velocities and metallicities similar to Pall are shown by the red solid circles in the left panel. The new Pal1 candidate stars, and Stars I and II, are selected as those closest to the isochrones. Star F is denoted by an empty red circle because it is flagged by SDSS with unreliable photometric magnitudes ("too few detection to be deblended").

mouth Stellar Evolution Database (Dotter et al. 2008) are included with ages of 4,5 and 6 Gyrs, with the distance, reddening, and metallicity from Sarajedini et al. (2007), and adopting $[\alpha / \mathrm{Fe}]=0$. However, the APOGEE target selection provides Gunn ugriz and JHK magnitudes of the targets (Doi et al. 2010), requiring conversion to Johnson VI. We have adopted the calibration from Table 4 of Jordi et al. (2006) for Population I stars. ${ }^{1}$

The right panel in Fig. 2 shows the CMD of 47 Tuc and an isochrone generated from the Dartmouth Stellar Evolution Database (Dotter et al. 2008) with an age of 12.2 Gyr. The distance and metallicity are from Sarajedini et al. 2007, with $[\alpha / \mathrm{Fe}]=0.4$ and $\mathrm{E}(\mathrm{B}-\mathrm{V})=0^{2}$. Comparing the CMD of Pal1 to that of 47 Tuc in Fig. 2 clearly shows that Pal1 is younger and more sparsely populated than a typical globular cluster.

The $\mathrm{V}$ and I magnitudes from this transformation for Stars I and II are in good agreement with those from the Sarajedini et al. (2007); see Table 1. An additional five stars (Stars D, E, F, G and H) with radial velocities and metallicities consistent with Pal1 were selected from near the isochrones. We examine the stellar properties of these additional five stars below.

\footnotetext{
1 The uncertainties are determined in quadrature given the uncertainties for each color index listed in the APOGEE DR12 database and formulae by Jordi et al. (2006).

2 The reddening for 47 Tuc of $\mathrm{E}(\mathrm{B}-\mathrm{V})=0.055$ from Sarajedini et al. (2007) does not fit the turn-off well. When no reddening is applied, the fit is better (a lower reddening was similarly found by Schlafly \& Finkbeiner 2011, E(B-V)=0.03).
}

\section{STARS I AND II}

The stellar parameters for Stars I and II are shown in Table 1, from the optical analysis by Sakari et al. (2011), and the IR analysis of the APOGEE spectra through the ASPCAP pipeline. These two sets of results are in very poor agreement, with differences of $\Delta \mathrm{T}_{\mathrm{eff}} \sim 1000 \mathrm{~K}$ and $\Delta \log g \sim 1.0$, resulting in differences in $\Delta[\mathrm{Fe} / \mathrm{H}] \sim-0.4$.

In order to understand these differences, the individual visit spectra for these two stars are examined. There are 24 visits for Star I and 21 visits for Star II, with SNR > 6. We find a clear persistence problem in many of the spectra, in additional to some other effects such as poor flat fielding or telluric division problems, poor night sky line removal, and several cosmic ray hits.

\subsection{Removing Persistence}

Individual visits for Stars I and II were extracted from the APOGEE database. The alignment of each spectrum was compared to Arcturus, in order to check the radial velocity corrections. Each visit was then broken into the three wavelength regions corresponding to the blue, green, and red detectors. Some of APOGEE's detectors suffered from persistence, which is the contamination of a spectrum by remnants of the previous exposure. The persistence problem is worse on the blue chip (1.514-1.581 $\mu$ ), see Fig. 3. We remove the portion of the spectrum coming from the blue chip detector for any visit that shows persistence. Occasionally it was also necessary to remove the green chip spectrum - we suspect that the green chip itself does not have the persistence problem, but that the data reduction processing of the visit induces a flat fielding problem when persistence is bad on the blue chip. After this process, the remaining spec- 
Table 1. DR12 ASPCAP results for members and candidates of Pal1

\begin{tabular}{|c|c|c|c|c|c|c|c|c|c|c|c|}
\hline & APOGEE ID & $\begin{array}{c}\mathrm{RA} \\
(\mathrm{deg})\end{array}$ & $\begin{array}{l}\text { Dec } \\
(\operatorname{deg})\end{array}$ & $\begin{array}{c}\mathrm{RV} \\
\left(\mathrm{kms}^{-1}\right)\end{array}$ & $\begin{array}{l}\mathrm{T}_{\text {eff }} \\
(\mathrm{K})\end{array}$ & $\begin{array}{l}\log g \\
(\operatorname{dex})\end{array}$ & $\begin{array}{c}{[\mathrm{Fe} / \mathrm{H}]} \\
(\mathrm{dex})\end{array}$ & $\begin{array}{c}{[\alpha / \mathrm{Fe}]} \\
(\mathrm{dex})\end{array}$ & $\begin{array}{c}\mathrm{V} \\
(\mathrm{mag})\end{array}$ & $\begin{array}{c}\mathrm{I} \\
(\mathrm{mag})\end{array}$ & $(\mathrm{S} / \mathrm{N})$ \\
\hline Star D & $2 \mathrm{M} 03100079+7853325$ & 47.503 & 78.892 & -84.2 & 4957.7 & 2.69 & -0.3 & 0.1 & 15.086 & 13.861 & 85.3 \\
\hline Star E & $2 \mathrm{M} 04023010+7935181$ & 60.625 & 79.588 & -78.4 & 4231.1 & 1.49 & -0.7 & 0.1 & 14.522 & 13.664 & 151.2 \\
\hline Star F & $2 \mathrm{M} 03354183+7841453$ & 53.924 & 78.696 & -84.9 & 4847.7 & 2.56 & -0.3 & 0.1 & 13.386 & 11.266 & 377.9 \\
\hline Star G & $2 \mathrm{M} 03070369+7933134$ & 46.765 & 79.554 & -62.0 & 4564.0 & 3.05 & -0.5 & 0.2 & 16.955 & 15.562 & 68.7 \\
\hline Star H & $2 \mathrm{M} 03122767+7927416$ & 48.115 & 79.462 & -87.4 & 4856.8 & 2.75 & -0.3 & 0.1 & 15.163 & 13.854 & 156.6 \\
\hline Star I & $2 \mathrm{M} 03332183+7935382$ & 53.341 & 79.594 & -75.2 & 5710.9 & 3.47 & -0.2 & 0.1 & 16.705 & 15.461 & 83.6 \\
\hline Star II & $2 \mathrm{M} 03332960+7934162$ & 53.373 & 79.571 & -75.3 & 5602.4 & 3.22 & -0.1 & 0.1 & 16.840 & 15.618 & 67.4 \\
\hline Star I & (Sakari et al. 2011) & 53.341 & 79.589 & -77.2 & 4800.0 & 2.27 & -0.61 & 0.01 & 16.705 & 15.459 & 15 \\
\hline Star II & (Sakari et al. 2011) & 53.373 & 79.571 & -78.0 & 4750.0 & 2.33 & -0.61 & -0.10 & 16.675 & 15.618 & 15 \\
\hline
\end{tabular}
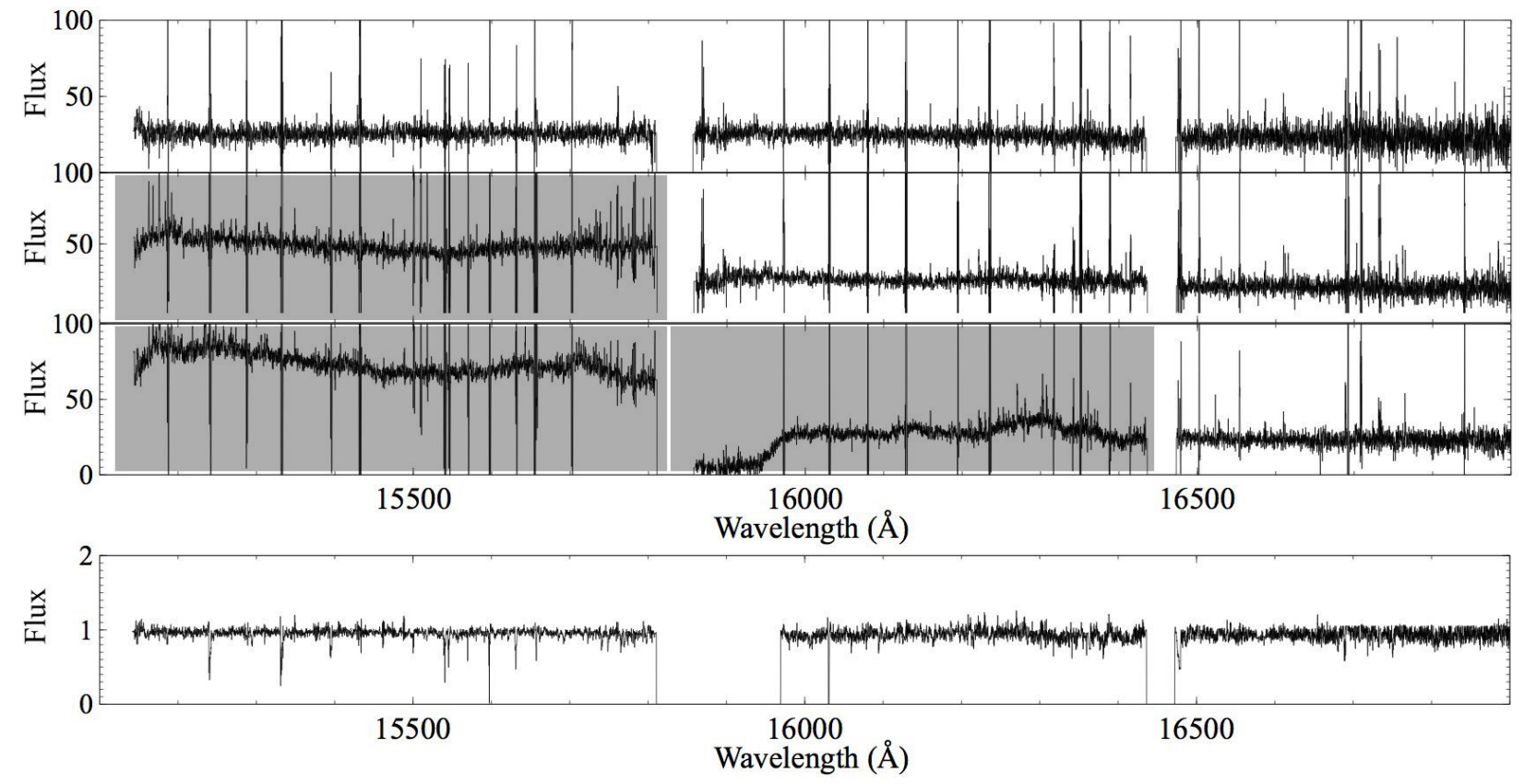

Figure 3. The top three panels are a sample of the spectra with no persistence problems (top), moderate persistence (middle), and strong persistence (or other flat fielding problems; bottom). All chips that would be removed in our analysis are shaded. The lower panel shows the final spectra after continuum normalization (see text) and removing sky lines

tra from each visit are co-added, i.e., only the non-persistent spectra from the blue, green, and red regions are kept for our analysis.

The non-persistent regions of each visit were combined to create the full wavelength range visits, and the cleaned visits were median-combined using IRAF. The final combined spectra for Stars I and II tend to have fewer green spectra than red, and fewer blue than either. This results in a lower SNR for the green than red spectrum, and lowest SNR for the blue spectrum. These spectra were then normalized with a Legendre polynomial (order $=8$ ), followed by a k-sigma clipping routine (see Venn et al. 2012), and sky lines are removed. These steps are illustrated in Fig. 3. Since these stars are moderately metal-poor, we found this normalization method to be sufficient for our purposes, but we caution that this is not the same as that used by the ASPCAP pipeline. Stars G and $\mathrm{H}$ also have significant persistence on their spectra. We have cleaned them similar to Stars I and II. Stars D, E and F did not have significant persistence problems. These gave us an opportunity to use and test ASPCAP on the original spectra in the APOGEE database.

In Fig. 4, a portion of the cleaned and combined spectra of our Pal1 members to that of Arcturus are compared. APOGEE spectra have $R=22,500$ whereas the Arcturus spectrum from (Hinkle et al. 2003) was convolved with a Gaussian profile to match the lower resolution and has $\mathrm{R}=24,000$.

Star G shows broader lines than Arcturus and the other spectra in our sample, which suggests that it is a dwarf star ${ }^{3}$.

In Fig. 4, the $\mathrm{CN}, \mathrm{OH}, \mathrm{Mg} \mathrm{I}, \mathrm{Al} \mathrm{I}, \mathrm{Si}$ I, and Fe I features in our candidate spectra are highlighted and compared to the Arcturus spectrum. Stars I and II exhibit weaker spectral lines for these species than Arcturus, which can be

3 The newest APOGEE DR13 grids for dwarfs include rotation models and therefore $\log g$ of Star G is removed in the new data release, which supports our claim that Star G is a dwarf star. 
Table 2. Details of each visit for members and candidates of Pal1

\begin{tabular}{|c|c|c|c|c|c|c|}
\hline Visit \# & Visit ID & $\begin{array}{l}\text { Heliocentric Velocity } \\
\qquad\left(\mathrm{kms}^{-1}\right)\end{array}$ & Chip A & Chip B & Chip C & $\mathrm{S} / \mathrm{N}$ \\
\hline \multicolumn{7}{|l|}{ Star I } \\
\hline 1 & 5283-55816-050 & -73.8 & $\mathrm{P}$ & $\checkmark$ & $\checkmark$ & 10.3 \\
\hline 2 & $5282-55822-203$ & -74.8 & $\checkmark$ & $\checkmark$ & $\checkmark$ & 8.5 \\
\hline 3 & $5283-55823-050$ & -71.9 & $\mathrm{P}$ & $\mathrm{P}$ & $\checkmark$ & 10.3 \\
\hline 4 & $5282-55823-203$ & -73.7 & $\checkmark$ & $\checkmark$ & $\checkmark$ & 15.3 \\
\hline 5 & $5282-55841-161$ & -76.0 & $\checkmark$ & $\checkmark$ & $\checkmark$ & 13.6 \\
\hline 6 & 5283-55843-050 & -73.1 & $\mathrm{P}$ & $\checkmark$ & $\checkmark$ & 8.3 \\
\hline 7 & $5283-55873-056$ & -77.2 & $\mathrm{P}$ & $\checkmark$ & $\checkmark$ & 8.2 \\
\hline 8 & 5283-55874-056 & -75.2 & $\mathrm{P}$ & $\checkmark$ & $\checkmark$ & 15.0 \\
\hline 9 & 5283-55905-053 & -76.5 & $\mathrm{P}$ & $\mathrm{P}$ & $\checkmark$ & 13.6 \\
\hline 10 & 5283-55906-053 & -75.7 & $\mathrm{P}$ & $\checkmark$ & $\checkmark$ & 6.5 \\
\hline 11 & 6246-56263-046 & - & $\mathrm{P}$ & $\mathrm{P}$ & $\mathrm{F}$ & 9.7 \\
\hline 12 & 6247-56264-053 & -76.6 & $\mathrm{P}$ & $\checkmark$ & $\checkmark$ & 9.8 \\
\hline 13 & $6247-56283-044$ & -76.7 & $\mathrm{P}$ & $\mathrm{P}$ & $\checkmark$ & 7.3 \\
\hline 14 & 6363-56284-183 & -75.0 & $\checkmark$ & $\checkmark$ & $\checkmark$ & 9.8 \\
\hline 15 & 6364-56285-047 & -76.7 & $\mathrm{P}$ & $\mathrm{P}$ & $\checkmark$ & 10.9 \\
\hline 16 & 6246-56539-039 & -75.3 & $\mathrm{P}$ & $\mathrm{P}$ & $\checkmark$ & 6.1 \\
\hline 17 & 6364-56561-038 & -72.8 & $\mathrm{P}$ & $\mathrm{P}$ & $\checkmark$ & 11.9 \\
\hline 18 & 6363-56583-232 & -76.4 & $\checkmark$ & $\mathrm{F}$ & $\checkmark$ & 16.8 \\
\hline 19 & 6364-56584-032 & - & $\mathrm{P}$ & $\mathrm{P}$ & $\mathrm{F}$ & 14.8 \\
\hline 20 & $6363-56587-183$ & -74.0 & $\checkmark$ & $\checkmark$ & $\checkmark$ & 14.7 \\
\hline 21 & $6365-56608-182$ & -76.0 & $\checkmark$ & $\checkmark$ & $\checkmark$ & 13.8 \\
\hline 22 & $6365-56642-200$ & -75.8 & $\checkmark$ & $\checkmark$ & $\checkmark$ & 12.0 \\
\hline 23 & 6366-56644-203 & -73.8 & $\checkmark$ & $\checkmark$ & $\checkmark$ & 12.5 \\
\hline 24 & 6365-56676-203 & -73.2 & $\checkmark$ & $\checkmark$ & $\checkmark$ & 13.4 \\
\hline \multicolumn{7}{|l|}{ Star II } \\
\hline 1 & 5283-55816-053 & -71.9 & $\mathrm{P}$ & $\checkmark$ & $\checkmark$ & 9.1 \\
\hline 2 & $5282-55822-204$ & -75.5 & $\checkmark$ & $\checkmark$ & $\checkmark$ & 7.1 \\
\hline 3 & $5283-55823-053$ & -71.4 & $\mathrm{P}$ & $\mathrm{P}$ & $\checkmark$ & 7.7 \\
\hline 4 & $5282-55823-204$ & -73.4 & $\checkmark$ & $\checkmark$ & $\checkmark$ & 12.9 \\
\hline 5 & $5282-55841-162$ & -75.1 & $\checkmark$ & $\checkmark$ & $\checkmark$ & 12.4 \\
\hline 6 & $5283-55843-053$ & -71.1 & $\mathrm{P}$ & $\checkmark$ & $\checkmark$ & 8.4 \\
\hline 7 & 5283-55873-059 & -73.0 & $\mathrm{P}$ & $\checkmark$ & $\checkmark$ & 7.2 \\
\hline 8 & 5283-55874-059 & -73.7 & $\mathrm{P}$ & $\mathrm{P}$ & $\checkmark$ & 13.8 \\
\hline 9 & 5283-55905-050 & -74.4 & $\mathrm{P}$ & $\mathrm{P}$ & $\checkmark$ & 11.0 \\
\hline 10 & $6246-56263-047$ & - & RV & RV & RV & 7.0 \\
\hline 11 & 6247-56264-049 & -76.2 & $\mathrm{P}$ & $\checkmark$ & $\checkmark$ & 9.1 \\
\hline 12 & $6247-56283-048$ & - & RV & RV & RV & 9.1 \\
\hline 13 & 6363-56284-182 & -72.8 & $\checkmark$ & $\mathrm{F}$ & $\mathrm{O}$ & 8.9 \\
\hline 14 & 6364-56285-044 & -71.6 & $\mathrm{P}$ & $\mathrm{P}$ & $\checkmark$ & 7.2 \\
\hline 15 & 6364-56561-041 & - & RV & RV & RV & 6.8 \\
\hline 16 & $6363-56583-233$ & -74.9 & $\checkmark$ & $\mathrm{F}$ & $\mathrm{O}$ & 13.5 \\
\hline 17 & 6363-56587-182 & -72.1 & $\checkmark$ & $\checkmark$ & $\checkmark$ & 11.8 \\
\hline 18 & $6365-56608-181$ & -73.9 & $\checkmark$ & $\checkmark$ & $\checkmark$ & 12.0 \\
\hline 19 & $6365-56642-199$ & 0 & $\checkmark$ & $\checkmark$ & $\checkmark$ & 10.0 \\
\hline 20 & 6366-56644-059 & -75.0 & $\mathrm{P}$ & $\mathrm{P}$ & $\checkmark$ & 11.2 \\
\hline 21 & 6365-56676-204 & -72.2 & $\checkmark$ & $\checkmark$ & $\mathrm{O}$ & 11.6 \\
\hline \multicolumn{7}{|l|}{ Star D } \\
\hline 1 & 5282-55815-010 & -66.1 & $\mathrm{P}$ & $\checkmark$ & $\checkmark$ & 5.4 \\
\hline 2 & $5282-55822-010$ & -82.6 & $\checkmark$ & $\checkmark$ & $\checkmark$ & 21.0 \\
\hline 3 & $5282-55823-010$ & -83.8 & $\checkmark$ & $\checkmark$ & $\checkmark$ & 35.8 \\
\hline 4 & $5282-55841-004$ & -87.7 & $\checkmark$ & $\checkmark$ & $\checkmark$ & 40.6 \\
\hline \multicolumn{7}{|l|}{ Star E } \\
\hline 1 & 6365-56608-154 & -78.5 & $\mathrm{P}$ & $\checkmark$ & $\checkmark$ & 68.2 \\
\hline 2 & $6365-56642-165$ & -78.5 & $\checkmark$ & $\checkmark$ & $\checkmark$ & 61.6 \\
\hline 3 & 6365-56676-16 & -78.2 & $\checkmark$ & $\checkmark$ & $\checkmark$ & 71.7 \\
\hline \multicolumn{7}{|l|}{ Star F } \\
\hline 1 & $6247-56264-148$ & -84.8 & $\checkmark$ & $\checkmark$ & $\checkmark$ & 165.2 \\
\hline 2 & 6247-56283-093 & -84.8 & $\checkmark$ & $\checkmark$ & $\checkmark$ & 170.5 \\
\hline 3 & 6247-56541-099 & -85.0 & $\checkmark$ & $\checkmark$ & $\checkmark$ & 88.4 \\
\hline 4 & $6247-56542-099$ & -85.1 & $\checkmark$ & $\checkmark$ & $\checkmark$ & 110.3 \\
\hline
\end{tabular}


Table 2 - continued

\begin{tabular}{|c|c|c|c|c|c|c|}
\hline Visit \# & Visit ID & $\begin{array}{c}\text { Heliocentric Velocity } \\
\left(\mathrm{kms}^{-1}\right)\end{array}$ & Chip A & Chip B & Chip C & $\mathrm{S} / \mathrm{N}$ \\
\hline \multicolumn{7}{|l|}{ Star G } \\
\hline 1 & $5282-55822-218$ & -64.0 & $\checkmark$ & $\checkmark$ & $\checkmark$ & 7.2 \\
\hline 2 & $5282-55823-218$ & -63.4 & $\checkmark$ & $\checkmark$ & $\checkmark$ & 12.9 \\
\hline 3 & $5282-55841-212$ & -63.9 & $\checkmark$ & $\checkmark$ & $\checkmark$ & 9.5 \\
\hline 4 & 5283-55816-013 & -57.2 & $\mathrm{P}$ & $\checkmark$ & $\checkmark$ & 7.3 \\
\hline 5 & 5283-55823-019 & -58.4 & $\mathrm{P}$ & $\checkmark$ & $\checkmark$ & 8.5 \\
\hline 6 & 5283-55843-013 & -56.9 & $\mathrm{P}$ & $\checkmark$ & $\checkmark$ & 8.2 \\
\hline 7 & 5283-55873-013 & -61.2 & $\mathrm{P}$ & $\checkmark$ & $\checkmark$ & 6.4 \\
\hline 8 & 5283-55874-013 & 61.7 & $\mathrm{P}$ & $\checkmark$ & $\checkmark$ & 13.1 \\
\hline 9 & 5283-55905-013 & -64.9 & $\mathrm{P}$ & $\checkmark$ & $\checkmark$ & 11.2 \\
\hline 10 & 5283-55906-013 & -63.2 & $\mathrm{P}$ & $\checkmark$ & $\checkmark$ & 5.4 \\
\hline 11 & 6246-56263-013 & -63.1 & $\mathrm{P}$ & $\checkmark$ & $\checkmark$ & 10.4 \\
\hline 12 & 6246-56282-019 & -68.0 & $\mathrm{P}$ & $\checkmark$ & $\checkmark$ & 5.9 \\
\hline 13 & $6246-56318-013$ & -72.5 & $\mathrm{P}$ & $\checkmark$ & $\checkmark$ & 5.3 \\
\hline 14 & 6246-56539-013 & -60.7 & $\mathrm{P}$ & $\checkmark$ & $\checkmark$ & 5.8 \\
\hline 15 & $6247-56264-216$ & -62.4 & $\checkmark$ & $\checkmark$ & $\checkmark$ & 11.1 \\
\hline 16 & $6247-56283-216$ & -45.1 & $\checkmark$ & $\checkmark$ & $\checkmark$ & 11.7 \\
\hline 17 & $6247-56541-211$ & -60.5 & $\checkmark$ & $\checkmark$ & $\checkmark$ & 7.5 \\
\hline 18 & $6247-56542-211$ & -63.1 & $\checkmark$ & $\checkmark$ & $\checkmark$ & 8.6 \\
\hline 19 & 6363-56284-013 & -68.1 & $\mathrm{P}$ & $\checkmark$ & $\checkmark$ & 6.4 \\
\hline 20 & 6363-56583-014 & -63.6 & $\mathrm{P}$ & $\checkmark$ & $\checkmark$ & 10.6 \\
\hline 21 & 6363-56587-013 & -62.6 & $\mathrm{P}$ & $\checkmark$ & $\checkmark$ & 9.5 \\
\hline 22 & 6364-56285-018 & -65.3 & $\mathrm{P}$ & $\checkmark$ & $\checkmark$ & 8.2 \\
\hline 23 & 6364-56561-019 & -59.9 & $\mathrm{P}$ & $\checkmark$ & $\checkmark$ & 9.6 \\
\hline 24 & 6364-56584-013 & -63.2 & $\mathrm{P}$ & $\checkmark$ & $\checkmark$ & 9.5 \\
\hline 25 & 6365-56608-013 & -64.1 & $\mathrm{P}$ & $\checkmark$ & $\checkmark$ & 9.1 \\
\hline 26 & 6365-56642-018 & -60.9 & $\mathrm{P}$ & $\checkmark$ & $\checkmark$ & 8.9 \\
\hline 27 & 6365-56676-018 & -65.8 & $\mathrm{P}$ & $\checkmark$ & $\mathrm{O}$ & 8.7 \\
\hline 28 & 6366-56644-013 & -62.2 & $\mathrm{P}$ & $\checkmark$ & $\checkmark$ & 8.2 \\
\hline \multicolumn{7}{|l|}{ Star H } \\
\hline 1 & $5282-55815-214$ & -87.7 & $\checkmark$ & $\checkmark$ & $\checkmark$ & 6.1 \\
\hline 2 & $5282-55822-214$ & -87.6 & $\checkmark$ & $\checkmark$ & $\checkmark$ & 26.5 \\
\hline 3 & $5282-55823-214$ & -87.4 & $\checkmark$ & $\checkmark$ & $\checkmark$ & 45.5 \\
\hline 4 & $5282-55841-232$ & -87.4 & $\checkmark$ & $\checkmark$ & $\checkmark$ & 40.4 \\
\hline 5 & 5283-55816-016 & -87.5 & $\mathrm{P}$ & $\checkmark$ & $\checkmark$ & 25.3 \\
\hline 6 & 5283-55823-022 & -87.0 & $\mathrm{P}$ & $\checkmark$ & $\checkmark$ & 33.9 \\
\hline 7 & 5283-55843-016 & -86.8 & $\mathrm{P}$ & $\checkmark$ & $\checkmark$ & 30.4 \\
\hline 8 & 5283-55873-016 & 86.8 & $\checkmark$ & $\checkmark$ & $\checkmark$ & 26.7 \\
\hline 9 & 5283-55874-016 & -87.3 & $\mathrm{P}$ & $\checkmark$ & $\checkmark$ & 47.5 \\
\hline 10 & 5283-55905-015 & -87.7 & $\mathrm{P}$ & $\checkmark$ & $\checkmark$ & 35.4 \\
\hline 11 & 5283-55906-015 & -87.5 & $\mathrm{P}$ & $\checkmark$ & $\checkmark$ & 17.1 \\
\hline
\end{tabular}

Note. $\mathrm{P}=$ persistence, $\mathrm{F}=$ flat problems, $\mathrm{RV}=$ incorrect $\mathrm{RV}, \mathrm{O}=$ other problems related to SNR or large noise spikes or poor night sky line removal..

attributed to their higher surface temperatures. The aforementioned line broadening observed in Star G is present in these spectral ranges as well.

\section{NEW STELLAR ANALYSES}

We have carried out a new analysis for all of the stars that may be members of Pal1 based on the DR12 data. This includes those stars that have a persistence problem, but also those that do not so that we treat the data for all of these objects in a similar way. New stellar parameters are determined, initially from optical and IR photometry using both the Casagrande et al. (2010) and Ramírez \& Meléndez (2005), colour-temperature relationships. Temperatures and bolometric corrections are determined from the unweighted average of four colours: (B-V), (V-I), (V-K), and (J-K), adopting the metallicity and cluster distance for Pal1 from Sarajedini et al. (2007). Reddening estimates are from Schlafly \& Finkbeiner (2011). Surface gravities are determined photometrically as in Venn et al. (2012), after adopting a cluster turn-off mass of $\mathrm{MA}=1.14 \mathrm{M}_{\odot}$ (Sakari et al. 2011) corresponding to its young age, such that:

$\log g=4.44+\log (M A)+4 \log \left(\frac{T_{\mathrm{eff}}}{5790}\right)+0.4\left(M_{\mathrm{bol}}-4.75\right)$

The $\mathrm{T}_{\text {eff }}$ values determined from the two different colortemperature calibrations were in excellent agreement for all of the candidates, with the exception of Star F. For this one star, the temperatures differed by $\Delta \mathrm{T}_{\text {eff }} \sim 1200 \mathrm{~K}$ (see Table 3). The temperature from Casagrande et al. (2010) is much higher, and inconsistent with the position of this 

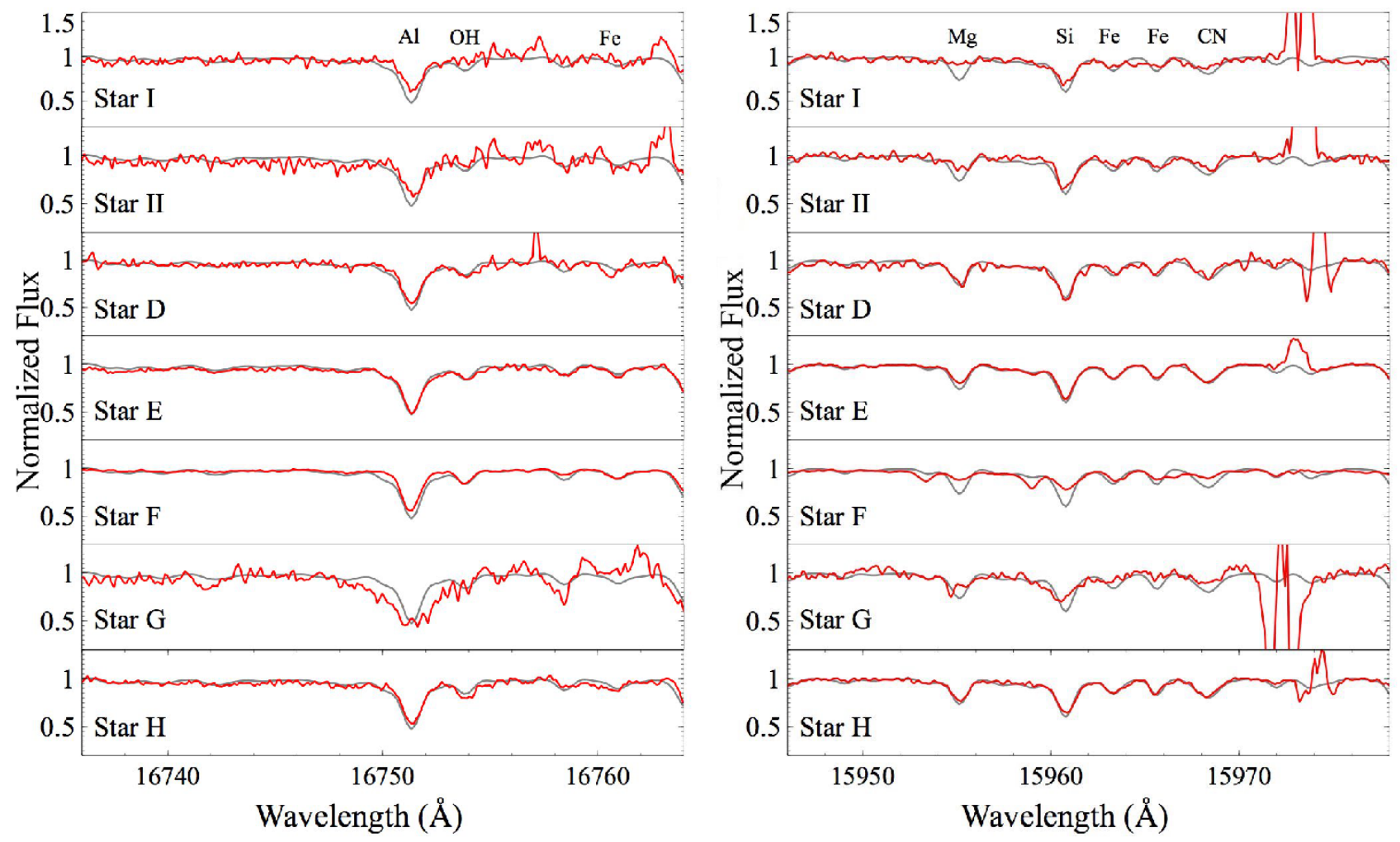

Figure 4. Comparing Pal1 member and candidate spectra (red) to Arcturus (grey). All spectra have been shifted to the Arcturus wavelength scale. Lines of Fe I, Mg I, Al I, Si I, OH and CN are labelled.

Table 3. Photometric Stellar Parameters

\begin{tabular}{lrrrrrr}
\hline & $\begin{array}{r}\mathrm{T}_{\boldsymbol{b} v} \\
(\mathrm{~K})\end{array}$ & $\begin{array}{r}\mathrm{T}_{\boldsymbol{v} \boldsymbol{i}} \\
(\mathrm{K})\end{array}$ & $\begin{array}{r}\mathrm{T}_{\boldsymbol{v} \boldsymbol{k}} \\
(\mathrm{K})\end{array}$ & $\begin{array}{r}\mathrm{T}_{\boldsymbol{j} \boldsymbol{k}} \\
(\mathrm{K})\end{array}$ & $\begin{array}{r}\mathrm{T}_{\text {eff }} \\
(\mathrm{K})\end{array}$ & $\log g$ \\
\hline Star I & 4930 & 4939 & 4872 & 4481 & 4806 & 2.27 \\
Star II & 5032 & 5015 & 4887 & - & 4978 & 2.33 \\
Star D & 4611 & 4635 & 4582 & 4660 & 4622 & 1.55 \\
Star E & 4516 & 4554 & 4415 & - & 4495 & 1.22 \\
Star F & 4787 & - & - & 5199 & 4993 & 1.64 \\
Star F* & - & 3800 & 3934 & - & 3867 & 0.43 \\
Star G & 4620 & 4632 & 4775 & 4670 & 4674 & 2.28 \\
Star H & 4787 & 4805 & 4826 & 4847 & 4816 & 1.47 \\
\hline
\end{tabular}

Note. $\mathrm{T}_{\text {eff }}$ of Star $\mathrm{F}^{*}$ is calculated using Ramírez \& Meléndez (2005) calibration and the rest are computed using Casagrande et al. (2010) calibration.

star on the colour-magnitude diagram in Fig. 2; however, the position of Star F in Fig. 2 depends on a correct $V$ magnitude, which has been flagged in the SDSS database. Without further information on the V magnitude of Star F, we consider both temperatures in the discussion below. The difference between the $\log g$ values for two different distance moduli from Harris (1996, 2010 edition) and Sarajedini et al. (2007) is $\Delta \log g \sim 0.4$, which causes only small to negligible differences in our abundance results.

The APOGEE ASPCAP data analysis pipeline uses the least squares template fitting routine, FERRE (Prieto et al. 2006), which matches observed spectra to (renormalized) synthetic spectra from model atmospheres that have been run through the $1 \mathrm{D}$, LTE, spectrum synthesis code ASSET (Koesterke et al. 2008; Koesterke et al. 2009). FERRE simultaneously determines the stellar parameters, metallicities, and element abundance ratios for a given spectrum. We too have used FERRE ${ }^{4}$ for metallicities and chemical abundances, once where FERRE determines the stellar parameters and a second time where we adopt our photometrically determined stellar parameters (see Tables 4-6). To match the observed spectra to the synthetic spectra, it was necessary to resample the observations to be on the same wavelength scale. This caused the observations to have a slightly lower resolution than the original visits, and the combined spectra had a slightly larger spectral range. This resulted in observations of a few additional absorption lines ( $\mathrm{K}, \mathrm{Mn})$ that that are not in the APOGEE DR12 database.

For Stars I and II, Table 4 shows that the photometric stellar parameters yield chemical abundances and metallicities in excellent agreement with the optical analyses. This implies that persistence is a significant problem in the analysis of these two stars in the DR12 data release (also see discussion of the DR13 data in Section 6.4). This further implies that the analysis of some stars in the APOGEE database can still be improved using the APOGEE spectra themselves.

${ }^{4}$ FERRE at Github: https://github.com/jobovy/apogee. 
Table 4. Different Properties of the candidates using FERRE

\begin{tabular}{|c|c|c|c|c|c|}
\hline & DR12 & DR13 & FERRE ${ }^{*}$ & Photom ${ }^{*}$ & Sakari et al. (2011) \\
\hline \multicolumn{6}{|l|}{ Star I } \\
\hline $\mathrm{T}_{\text {eff }}(\mathrm{K})$ & 5711 & 5203 & $4806 \pm 92$ & $4806 \pm 218$ & $4800 \pm 70$ \\
\hline $\log g$ & 3.5 & 2.7 & 2.8 & $2.3 \pm 0.2$ & $2.27 \pm 0.15$ \\
\hline$[\mathrm{FeI} / \mathrm{H}]$ & -0.2 & -0.4 & $-0.8 \pm 0.1$ & $-0.6 \pm 0.0$ & $-0.61 \pm 0.08$ \\
\hline$[\alpha / \mathrm{Fe}]$ & 0.1 & 0.2 & - & $0.1 \pm 0.1$ & $0.00 \pm 0.00$ \\
\hline$[\mathrm{C} / \mathrm{Fe}]$ & -0.2 & - & $0.5 \pm 0.2$ & $<0.1$ & - \\
\hline$[\mathrm{Ca} / \mathrm{Fe}]$ & 0.4 & -0.4 & $0.3 \pm 0.2$ & $0.2 \pm 0.1$ & $0.16 \pm 0.16$ \\
\hline$[\mathrm{S} / \mathrm{Fe}]$ & -0.3 & - & $0.6 \pm 0.2$ & $0.2 \pm 0.1$ & - \\
\hline$[\mathrm{O} / \mathrm{Fe}]$ & 1.0 & 0.8 & $0.2 \pm 0.4$ & $0.4 \pm 0.1$ & $<0.82$ \\
\hline$[\mathrm{Mg} / \mathrm{Fe}]$ & -0.1 & 0.1 & $-0.3 \pm 0.3$ & $-0.1 \pm 0.2$ & $-0.11 \pm 0.20$ \\
\hline$[\mathrm{Mn} / \mathrm{Fe}]$ & 0.0 & -0.2 & $0.9 \pm 0.1$ & $-0.1 \pm 0.1$ & - \\
\hline$[\mathrm{Si} / \mathrm{Fe}]$ & 0.3 & 0.1 & $-0.1 \pm 0.1$ & $0.1 \pm 0.2$ & $0.24 \pm 0.24$ \\
\hline$[\mathrm{Al} / \mathrm{Fe}]$ & - & - & $0.1 \pm 0.1$ & $0.1 \pm 0.2$ & - \\
\hline$[\mathrm{K} / \mathrm{Fe}]$ & - & - & $0.1 \pm 0.1$ & $0.1 \pm 0.1$ & - \\
\hline \multicolumn{6}{|l|}{ Star II } \\
\hline $\mathrm{T}_{\mathrm{eff}}(\mathrm{K})$ & 5602 & 4886 & $4936 \pm 92$ & $4978 \pm 79$ & $4750 \pm 135$ \\
\hline $\log g$ & 3.2 & 2.3 & 2.7 & $2.3 \pm 0.2$ & $2.33 \pm 0.15$ \\
\hline$[\mathrm{FeI} / \mathrm{H}]$ & -0.1 & -0.5 & $-0.5 \pm 0.2$ & $-0.6 \pm 0.0$ & $-0.61 \pm 0.08$ \\
\hline$[\alpha / \mathrm{Fe}]$ & 0.1 & 0.2 & - & $0.1 \pm 0.1$ & $-0.10 \pm 0.00$ \\
\hline$[\mathrm{C} / \mathrm{Fe}]$ & -0.3 & - & $0.1 \pm 0.2$ & $<0.2$ & - \\
\hline$[\mathrm{Ca} / \mathrm{Fe}]$ & 0.3 & -0.3 & $0.4 \pm 0.2$ & $0.2 \pm 0.3$ & $-0.04 \pm 0.22$ \\
\hline$[\mathrm{S} / \mathrm{Fe}]$ & -0.6 & - & $0.0 \pm 0.2$ & $0.2 \pm 0.1$ & - \\
\hline$[\mathrm{O} / \mathrm{Fe}]$ & -0.1 & 0.7 & $0.1 \pm 0.1$ & $0.2 \pm 0.1$ & $<0.32$ \\
\hline$[\mathrm{Mg} / \mathrm{Fe}]$ & -0.6 & 0.0 & $-0.2 \pm 0.2$ & $-0.1 \pm 0.1$ & $-0.13 \pm 0.30$ \\
\hline$[\mathrm{Mn} / \mathrm{Fe}]$ & 0.0 & -0.1 & $0.5 \pm 0.1$ & $-0.1 \pm 0.1$ & $-0.16 \pm 0.36$ \\
\hline$[\mathrm{Si} / \mathrm{Fe}]$ & 0.2 & 0.2 & $0.1 \pm 0.2$ & $0.2 \pm 0.1$ & $0.13 \pm 0.23$ \\
\hline$[\mathrm{Al} / \mathrm{Fe}]$ & 0.0 & - & $-0.3 \pm 0.1$ & $-0.1 \pm 0.1$ & - \\
\hline$[\mathrm{K} / \mathrm{Fe}]$ & -0.8 & - & $0.0 \pm 0.1$ & $0.2 \pm 0.1$ & - \\
\hline
\end{tabular}

* This use of "FERRE" is on our persistence cleaned spectra, allowing FERRE to simultaneously determine the stellar parameters and chemical abundances, whereas "Photom" uses our photometrically determined stellar parameters.

Table 5. Different Properties of the candidates using FERRE

\begin{tabular}{lrrrr}
\hline & DR12 & DR13 & FERRE $^{*}$ & Photom $^{*}$ \\
\hline Star D & & & & \\
& & & & \\
$\mathrm{T}_{\text {eff }}(\mathrm{K})$ & 4958 & 4843 & $4870 \pm 92$ & $4622 \pm 33$ \\
$\log g$ & 2.7 & 2.6 & $2.8 \pm 0.1$ & $1.6 \pm 0.2$ \\
{$[\mathrm{FeI} / \mathrm{H}]$} & -0.3 & -0.3 & $-0.4 \pm 0.1$ & $-0.6 \pm 0.0$ \\
{$[\boldsymbol{\alpha} / \mathrm{Fe}]$} & 0.1 & 0.1 & - & $0.1 \pm 0.1$ \\
{$[\mathrm{C} / \mathrm{Fe}]$} & 0.3 & - & $0.3 \pm 0.2$ & $0.1 \pm 0.1$ \\
{$[\mathrm{Ca} / \mathrm{Fe}]$} & 0.3 & 0.3 & $0.4 \pm 0.1$ & $0.2 \pm 0.2$ \\
{$[\mathrm{~S} / \mathrm{Fe}]$} & 0.1 & - & $0.1 \pm 0.1$ & $0.1 \pm 0.1$ \\
{$[\mathrm{O} / \mathrm{Fe}]$} & 0.0 & 0.5 & $-0.1 \pm 0.4$ & $0.3 \pm 0.1$ \\
{$[\mathrm{Mg} / \mathrm{Fe}]$} & 0.2 & 0.0 & $0.2 \pm 0.1$ & $-0.2 \pm 0.1$ \\
{$[\mathrm{Mn} / \mathrm{Fe}]$} & -0.2 & -0.1 & $0.4 \pm 0.1$ & $-0.1 \pm 0.1$ \\
{$[\mathrm{Si} / \mathrm{Fe}]$} & 0.2 & 0.2 & $0.1 \pm 0.1$ & $0.1 \pm 0.1$ \\
{$[\mathrm{Al} / \mathrm{Fe}]$} & -0.7 & - & $-0.6 \pm 0.1$ & $0.4 \pm 0.1$ \\
{$[\mathrm{~K} / \mathrm{Fe}]$} & -0.4 & - & $-0.2 \pm 0.1$ & $-0.2 \pm 0.1$
\end{tabular}

Star $\mathbf{E}$

$\begin{array}{lrrrr}\mathrm{T}_{\text {eff }} & 4231 & 4138 & 4168 \pm 92 & 4495 \pm 72 \\ \log g & 1.5 & 1.3 & 1.9 \pm 0.1 & 1.2 \pm 0.15\end{array}$


Table 5 - continued

\begin{tabular}{lrrrr}
\hline & DR12 & DR13 & FERRE $^{*}$ & Photom $^{*}$ \\
\hline$[\mathrm{FeI} / \mathrm{H}]$ & -0.7 & -0.6 & $-0.6 \pm 0.1$ & $-0.6 \pm 0.0$ \\
{$[\alpha / \mathrm{Fe}]$} & 0.1 & 0.1 & - & $0.2 \pm 0.1$ \\
{$[\mathrm{C} / \mathrm{Fe}]$} & 0.0 & - & $-0.1 \pm 0.0$ & $0.1 \pm 0.1$ \\
{$[\mathrm{Ca} / \mathrm{Fe}]$} & 0.0 & 0.1 & $0.0 \pm 0.1$ & $0.2 \pm 0.2$ \\
{$[\mathrm{~S} / \mathrm{Fe}]$} & 0.2 & - & $0.1 \pm 0.1$ & $0.2 \pm 0.5$ \\
{$[\mathrm{O} / \mathrm{Fe}]$} & -0.2 & 0.1 & $0.1 \pm 0.1$ & $0.5 \pm 0.1$ \\
{$[\mathrm{Mg} / \mathrm{Fe}]$} & -0.3 & 0.1 & $0.0 \pm 0.1$ & $0.3 \pm 0.1$ \\
{$[\mathrm{Mn} / \mathrm{Fe}]$} & 0.0 & -0.1 & $0.6 \pm 0.0$ & $0.0 \pm 0.2$ \\
{$[\mathrm{Si} / \mathrm{Fe}]$} & -0.2 & 0.1 & $0.2 \pm 0.1$ & $0.0 \pm 0.1$ \\
{$[\mathrm{Al} / \mathrm{Fe}]$} & 0.2 & - & $0.3 \pm 0.1$ & $0.3 \pm 0.0$ \\
{$[\mathrm{~K} / \mathrm{Fe}]$} & 0.0 & - & $0.0 \pm 0.1$ & $0.0 \pm 0.1$
\end{tabular}

Star G

$\begin{array}{lrrrr}\mathrm{T}_{\text {eff }}(\mathrm{K}) & 4564 & 4472 & 4425 \pm 92 & 4674 \pm 70 \\ \log g & 3.1 & - & 4.6 \pm 0.1 & 2.3 \pm 0.2 \\ {[\mathrm{FeI} / \mathrm{H}]} & -0.5 & 0.0 & -0.2 \pm 0.1 & -0.6 \pm 0.0 \\ {[\boldsymbol{\alpha} / \mathrm{Fe}]} & 0.2 & 0.1 & - & - \\ {[\mathrm{C} / \mathrm{Fe}]} & 0.5 & - & 0.2 \pm 0.1 & <0.2 \\ {[\mathrm{Ca} / \mathrm{Fe}]} & - & 0.0 & 0.0 \pm 0.1 & - \\ {[\mathrm{S} / \mathrm{Fe}]} & 0.0 & - & -0.8 \pm 0.2 & - \\ {[\mathrm{O} / \mathrm{Fe}]} & 0.3 & 0.1 & 0.1 \pm 0.1 & 0.1 \pm 0.1 \\ {[\mathrm{Mg} / \mathrm{Fe}]} & 0.2 & 0.1 & 0.4 \pm 0.1 & 0.8 \pm 0.1 \\ {[\mathrm{Mn} / \mathrm{Fe}]} & -0.7 & -0.1 & 0.2 \pm 0.1 & -0.1 \pm 0.3 \\ {[\mathrm{Si} / \mathrm{Fe}]} & -0.0 & 0.2 & 0.3 \pm 0.1 & 0.2 \pm 0.1 \\ {[\mathrm{Al} / \mathrm{Fe}]} & - & - & 0.0 \pm- & <0.7 \\ {[\mathrm{~K} / \mathrm{Fe}]} & -0.1 & - & 0.2 \pm 0.2 & <0.7\end{array}$

Star $\mathbf{H}$

\begin{tabular}{lrrrr}
$\mathrm{T}_{\text {eff }}(\mathrm{K})$ & 4857 & 4800 & $4780 \pm 92$ & $4816 \pm 26$ \\
$\log g$ & 2.8 & 2.9 & $3.1 \pm 0.1$ & $1.5 \pm 0.2$ \\
{$[\mathrm{Fe} / \mathrm{H}]$} & -0.3 & -0.3 & $-0.3 \pm 0.1$ & $-0.4 \pm 0.0$ \\
{$[\alpha / \mathrm{Fe}]$} & 0.1 & 0.1 & - & $0.2 \pm 0.1$ \\
{$[\mathrm{C} / \mathrm{Fe}]$} & 0.2 & - & $-0.1 \pm 0.1$ & $0.2 \pm 0.1$ \\
{$[\mathrm{Ca} / \mathrm{Fe}]$} & 0.2 & 0.1 & $0.0 \pm 0.0$ & $0.1 \pm 0.0$ \\
{$[\mathrm{~S} / \mathrm{Fe}]$} & -0.2 & - & $0.4 \pm 0.1$ & $0.2 \pm 0.1$ \\
{$[\mathrm{O} / \mathrm{Fe}]$} & 0.1 & 0.3 & $0.1 \pm 0.2$ & $0.2 \pm 0.1$ \\
{$[\mathrm{Mg} / \mathrm{Fe}]$} & 0.2 & 0.2 & $0.1 \pm 0.1$ & $0.5 \pm 0.1$ \\
{$[\mathrm{Mn} / \mathrm{Fe}]$} & -0.2 & -0.1 & $0.3 \pm 0.0$ & $0.1 \pm 0.2$ \\
{$[\mathrm{Si} / \mathrm{Fe}]$} & 0.2 & 0.1 & $0.1 \pm 0.1$ & $0.1 \pm 0.1$ \\
{$[\mathrm{Al} / \mathrm{Fe}]$} & 0.0 & - & $-0.2 \pm-$ & $0.3 \pm 0.1$ \\
{$[\mathrm{~K} / \mathrm{Fe}]$} & -0.3 & - & $0.0 \pm 0.1$ & $0.0<$ \\
\hline
\end{tabular}

* This use of "FERRE" is on our persistence cleaned spectra, allowing FERRE to simultaneously determine the stellar parameters and chemical abundances, whereas "Photom" uses our photometrically determined stellar parameters.

Table 6. Different Properties of Star F

\begin{tabular}{lrrrrr}
\hline & DR12 & DR13 & FERRE $^{*}$ & Photom1 $^{*}$ & Photom2 $^{*}$ \\
\hline Star F & & & & & \\
& & & & & \\
$\mathrm{T}_{\text {eff }}(\mathrm{K})$ & 4848 & 4828 & $4875 \pm 92$ & $4993 \pm 291$ & $3867 \pm 95$ \\
$\log g$ & 2.6 & 2.5 & $3.2 \pm 0.1$ & $1.6 \pm 0.2$ & $0.4 \pm 0.2$ \\
{$[\mathrm{Fe} / \mathrm{H}]$} & -0.3 & -0.4 & $-0.8 \pm 0.1$ & $-0.6 \pm 0.1$ & $-1.4 \pm 0.1$ \\
{$[\alpha / \mathrm{Fe}]$} & 0.1 & 0.1 & - & $-0.3 \pm 0.3$ & - \\
{$[\mathrm{C} / \mathrm{Fe}]$} & -0.1 & - & $0.4 \pm 0.3$ & $0.3 \pm 0.1$ & $-0.1 \pm 0.1$
\end{tabular}


Table 6 - continued

\begin{tabular}{lrrrrr}
\hline & DR12 & DR13 & FERRE $^{*}$ & Photom1 $^{*}$ & Photom2 $^{*}$ \\
\hline$[\mathrm{Ca} / \mathrm{Fe}]$ & -0.1 & 0.0 & $0.0 \pm 0.1$ & $-0.3 \pm 0.1$ & - \\
{$[\mathrm{S} / \mathrm{Fe}]$} & 0.2 & - & $-0.1 \pm 0.2$ & $-0.4 \pm 0.1$ & $-0.2 \pm 0.1$ \\
{$[\mathrm{O} / \mathrm{Fe}]$} & 0.1 & 0.1 & $-0.1 \pm 0.3$ & $-0.2 \pm 0.2$ & $0.0 \pm 0.1$ \\
{$[\mathrm{Mg} / \mathrm{Fe}]$} & 0.0 & 0.1 & $0.2 \pm 0.1$ & $-0.3 \pm 0.2$ & $-0.5 \pm 0.1$ \\
{$[\mathrm{Mn} / \mathrm{Fe}]$} & 0.1 & 0.0 & $0.8 \pm 0.0$ & $0.3 \pm 0.2$ & $0.3 \pm 0.1$ \\
{$[\mathrm{Si} / \mathrm{Fe}]$} & 0.2 & 0.1 & $0.0 \pm 0.0$ & $-0.1 \pm 0.2$ & $0.0 \pm 0.1$ \\
{$[\mathrm{Al} / \mathrm{Fe}]$} & 0.2 & - & $0.6 \pm 0.0$ & $0.8 \pm 0.1$ & $0.8 \pm 0.1$ \\
{$[\mathrm{~K} / \mathrm{Fe}]$} & -0.1 & - & $-0.1 \pm 0.1$ & $0.1 \pm 0.1$ & $-0.2 \pm 0.1$ \\
\hline
\end{tabular}

* This use of "FERRE" is on our persistence cleaned spectra, allowing FERRE to simultaneously determine the stellar parameters and chemical abundances. For Star F, we found two very different temperatures depending on which set of photometric magnitudes were examined; see Table 3. Here we present the elemental abundances for each temperature.

\section{STELLAR ABUNDANCES}

The stellar parameters and chemical abundances for 10 elements have been redetermined in this paper for in a set of Pal1 members and candidates from persistence-cleaned APOGEE spectra. The results are shown in Tables 4-6, including the elements $\mathrm{C}, \mathrm{O}, \mathrm{Mg}, \mathrm{Al}, \mathrm{Si}, \mathrm{S}, \mathrm{K}, \mathrm{Ca}, \mathrm{Mn}$, and Fe (see Table A1 for log abundances of all detected lines).

The abundance uncertainties are calculated in two ways. When fewer than four lines are available, the error is taken as the standard deviation in $[\mathrm{Fe} / \mathrm{H}]$. When there are more than four lines, the measurement error is taken as the standard deviation divided by root of number of lines. For cases where either of these methods results in an error $<0.1 \mathrm{dex}$, an error of 0.1 dex is adopted since the best synthetic fits have been determined by eye.

A few elements require special notes:

- Titanium: Holtzman et al. (2015) show that the APOGEE (DR12) abundances do not reproduce the [Ti/Fe] trends seen for stars in the solar neighbourhood by Bensby et al. (2014). This difference is not currently understood, and therefore the ASPCAP titanium lines are to be treated with caution. Hawkins et al. (2016) suggested that the Ti line at $15837.8 \AA$, which is not included in the set adopted by ASPCAP, can be considered reliable. We did not use this line in our FERRE estimates.

- $[\alpha / \mathrm{Fe}]$ : We estimate a mean $[\alpha / \mathrm{Fe}]$ ratio by averaging the results for $\mathrm{Mg}, \mathrm{S}, \mathrm{Si}$ and $\mathrm{Ca}$ (not $\mathrm{O}$ due to the very noisy oxygen lines, and not $\mathrm{Ti}$ as discussed above).

Overall, the chemical abundances of Stars I and II are in a good agreement with the optical analysis by Sakari et al. (2011). Three candidate stars (Stars D, E, and G) have stellar parameters typical of red giants and metallicities of $[\mathrm{Fe} / \mathrm{H}]=-0.6$, when determined from the photometric parameters. These values are similar to the members in the core of Pal1. On the other hand, the chemistry of Star H is sufficiently different that it is a likely non-member.

Star F warrants special attention due to its position in the tidal tails of Pall. Two temperatures have been determined from the color-temperature calibrations for this star, based on its photometric uncertainties (see Table 3). When the cooler temperature is examined, then its metal- licity is significantly different from that of Pal1 such that it would be a non-member. However, if the hotter temperature is adopted, its stellar parameters are typical of a red giant, with a metallicity and chemical abundances that are similar to those of the members of Pall. Furthermore, with the hotter temperature, then Star $\mathrm{F}$ has a low $[\alpha / \mathrm{Fe}]$ that is consistent with the other members of Pal1. Its high $[\mathrm{Al} / \mathrm{Fe}]$, with slighly low $[\mathrm{Mg} / \mathrm{Fe}]$, is unusual for a star in Pall, unless Star F is, or has been contaminated by, an AGB star (e.g., Ventura \& D'Antona 2008).

\section{DISCUSSION}

Using the APOGEE database, we have re-examined the spectra for two known members of Pal1 and five new candidate members that are well away from the central region of this cluster. For each member and candidate star, all visits were examined and the blue chips of the spectra with persistence removed, then recombined the clean visits (see section 3.1 for more details). A new stellar analysis has been conducted using FERRE. The results for the cleaned spectra of Stars I and II are in excellent agreement with the optical analysis by Sakari et al. (2011), whereas the DR12 analyses based on the original spectra are not (see Table 1). The chemical abundance and stellar parameters of the candidates are shown in Table 4-6. The estimated $[\alpha / \mathrm{Fe}]$ ratios for Stars I and II are in good agreement with the optical results of Sakari et al. (2011). The Na I lines are too weak or noisy in most of the spectra for reliable determinations of $[\mathrm{Na} / \mathrm{Fe}]$, therefore we do not investigate the $\mathrm{Na}-\mathrm{O}$ anticorrelation.

\subsection{Tidal Tails of Pal1}

The position of the Pall candidates with respect to the tidal tails mapped out by Niederste-Ostholt et al. (2010) based on SDSS photometry are shown in Fig. 5. Their contour map is constructed from a probability-weighted star count map of Pall candidates from the CMD in the MSTO/MS region. The number of candidates per square arcmin can be determined as 0.856 at the centre of Pal1 and 0.050 above the background region. The positions of Stars I, II, and D-H are 


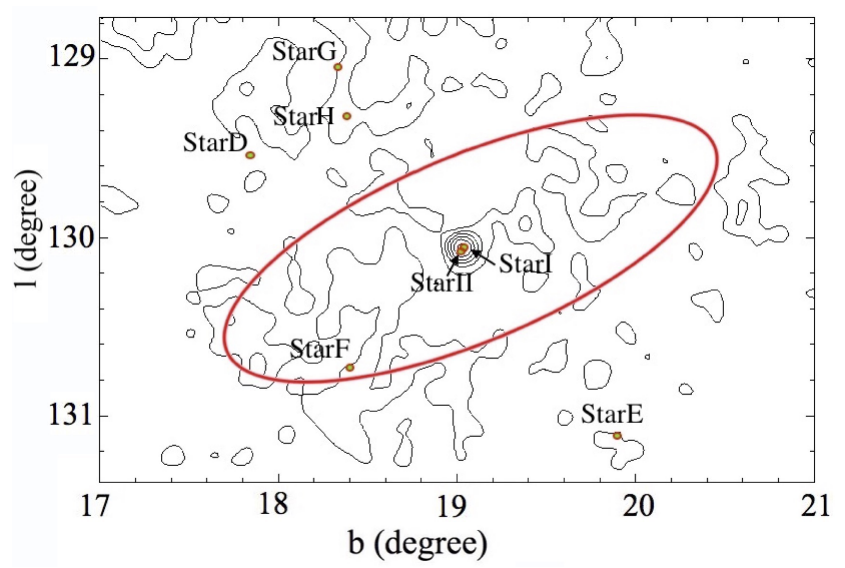

Figure 5. Position diagram of our Pall members and candidates relative to the SDSS stellar densities around Pal1. The red outline shows the position of the tails from Niederste-Ostholt et al. (2010). Our stars do not lie in those tails, except Star F.

shown relative to new isophots determined by M. Irwin from the same SDSS data in Fig. 5. A difference in the adopted bin sizes and isophot levels can suppress the apparent tidal features. Only one of these candidates, Star F, is coincident with the tidal tail found by Niederste-Ostholt et al. (2010).

Given the distance to Pal1 as $14.2 \mathrm{kpc}$ from the Sun (Sarajedini et al. 2007), and the angular separations of each star from its core, then the minimum distances of each star from the core of Pal1 range from $220.8 \mathrm{pc}$ (Star F) and 236.9 pc (Star H), to 294.6 pc (Star G), 319.8 pc (Star D), and $326.7 \mathrm{pc}$ (Star E). For these stars to have reached these distances over the lifetime of this cluster $(<6 \mathrm{Gyr})$ would have required ejection velocities $\leq 1 \mathrm{kms}^{-1}$. These velocities are not particularly large, therefore it is possible that if stars escape from Pall then they could be lurking at these angular separations.

\subsection{Membership Probability Analysis}

We examined the Besançon model (Robin et al. 2003) of the stellar populations in the Galaxy to evaluate membership probability of the new Pal1 candidates. The number of stars in the smooth Galactic halo in the direction of Pal1 are estimated based on similar limits in magnitude, colour, radial velocity, and metallicity (Fig. 6). To extract this simulated dataset, we run the model with the following selection criteria:

- an H-band range of 7 to 13.8 , comparable to the APOGEE target list.

- a distance interval from 0 to $50 \mathrm{kpc}$, to include most of both foreground and background stars.

- a 7 sq. deg. field of view, centred on Pal1 to match the SDSS field.

- The APOGEE database flags all non-giant stars as dwarfs. To directly compare the Besançon results with APOGEE, MS, WDs and T Tauri stars were removed from our Besançon model and only giant stars were taken into account.

These selection criteria result in 1124 total stars in

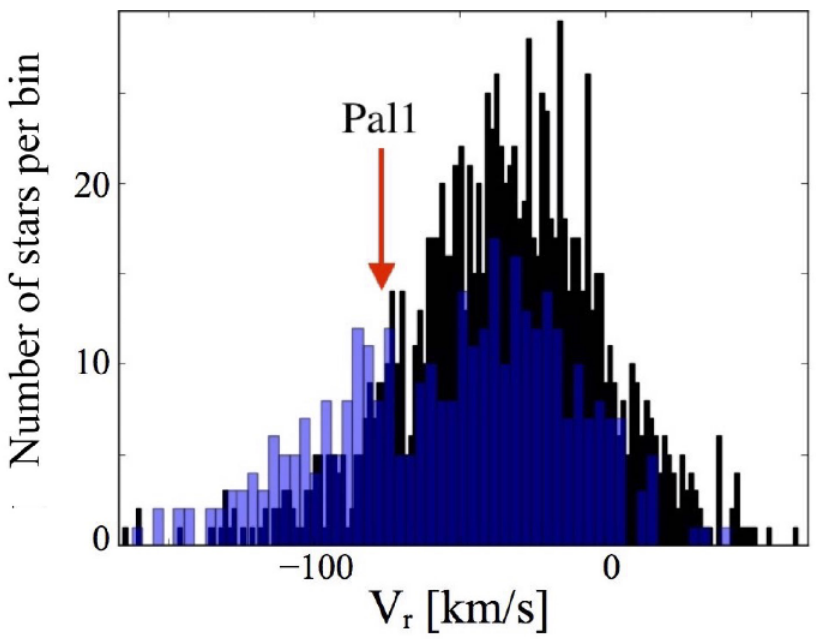

Figure 6. Histogram of the heliocentric radial velocities of APOGEE data (blue bars) and as determined from the Besançon model (Robin et al. 2003) in the direction of Pal 1 (black bars, renormalized). This suggests that our candidate Pal1 objects could be consistent with sampling of the smooth Galactic halo distribution.

the Besançon model. 129 (12\%) stars have radial velocities $\left(-75 \pm 15 \mathrm{kms}^{-1}\right)$ and metallicities $([\mathrm{Fe} / \mathrm{H}]=-0.6 \pm$ $0.4)$ similar to our parameters for the APOGEE search in the Pall field. These should be treated as field contaminants from the smooth halo distribution. In comparison, the SDSS/APOGEE Pal1 field contains 377 giants, of which 33 (9\%) have radial velocities and metallicities similar to Pall. Therefore, the Besançon model predicts a larger fraction of field contaminants $(12 \%)$ than observed in the APOGEE Pal1 field (9\%). This strongly implies that the APOGEE field is representative of the smooth halo, with no evidence for additional stars due to the Pal1 globular cluster.

It should be noted that APOGEE's Pall field is subject to observational placements, particularly in the fibre limitations. These include (1) crowding in the centre of Pal1 where the bonafide members are located, (2) that not all red giants can be observed simultaneously, and (3) that only $30 \%$ of the total number of good targets were observed.

A Monte Carlo approach was also used to randomly examine the potential for extracting Pall members from APOGEE Pal1 field. This was done by selecting $30 \%$ of stars from Besançon model to account for the APOGEE selections. For each sampling run, the fraction of field stars with our search criteria for Pal1 radial velocities and metallicities $\left(-75 \pm 15 \mathrm{kms}^{-1}\right.$ and $[\mathrm{Fe} / \mathrm{H}]=-0.6 \pm 0.4 \mathrm{dex}$, respectively) was calculated. 10000 runs were performed and the histogram of the distribution of corrected field contaminants is shown in Fig. 7. This histogram shows a well defined Gaussian distribution with a mean fraction of Pall contaminants of $0.12 \pm 0.02$.

Considering that number of stars in the RV and $[\mathrm{Fe} / \mathrm{H}]$ search criteria in the APOGEE Pal1 field yielded 33 out of 377 stars (or 9\%), we find that this is consistent with the predicted estimate from our Monte Carlo sampling of the Besançon smooth halo. 


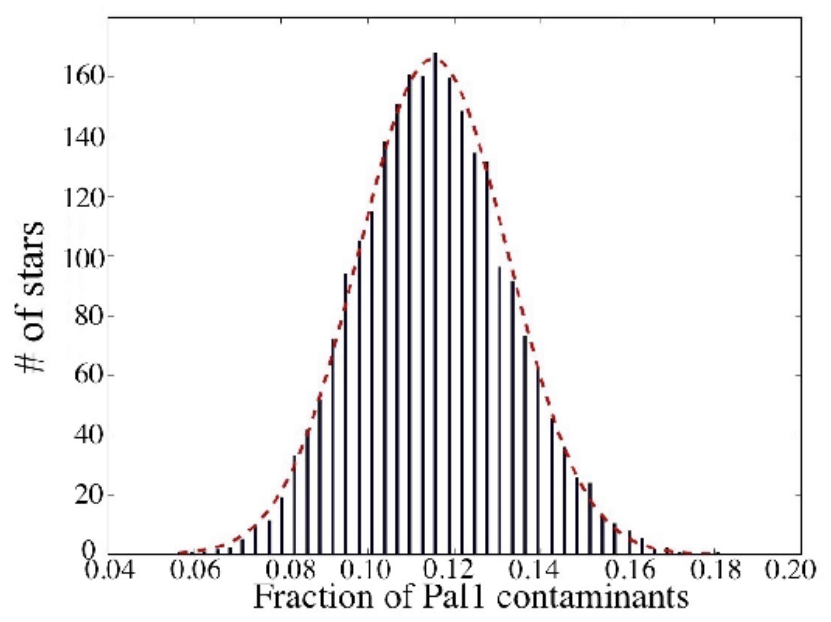

Figure 7. The histogram of the Gaussian distribution of Pal1 field contamination after 10000 runs in the Monte Carlo simulation. This plot suggests that $\sim 12 \%$ of stars in Pall field have radial velocities and metallicities comparable to our search criteria around the Pall core.

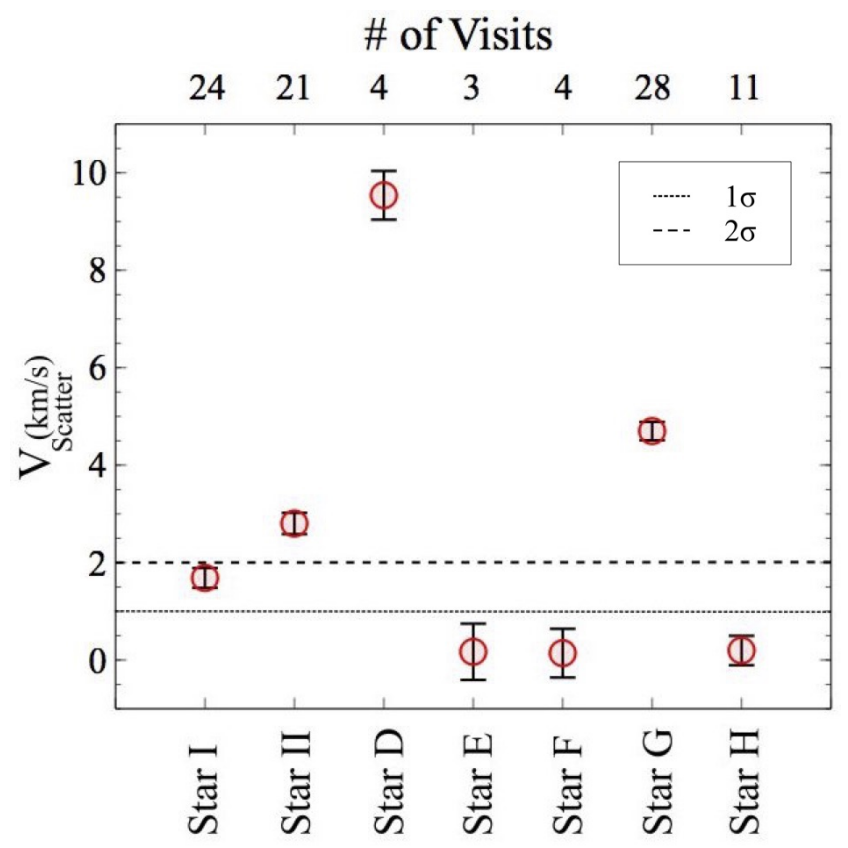

Figure 8. Velocity variation of the Pal1 members and candidates, with respect to number of visits for each star. When the scatter is $\geq 1 \mathrm{kms}^{-1}$ (dotted line), there is a high probability of a binary system. Error bars are $1 / \sqrt{\# \text { of visits. }}$

\subsection{Binarity?}

The velocity variation of the candidates are examined to find any evidence for a binarity, which could affect the stellar parameters analysis. The radial velocity variations for the two Pal1 members and for all of our candidate stars are shown in Fig. 8. Note that the y-axis represent the RV scatter of the candidates and is the standard deviation of all visits for each target. Nidever et al. (2015) has analysed a Plate-to-Plate
RV variation analysis for the APOGEE stars and found that the RVs in the APOGEE database are very stable as the rms scatter is $\sigma=0.044 \mathrm{kms}^{-1}$. They suggest that stars with RV scatter of greater than $1 \mathrm{kms}^{-1}$ have uncertainty much larger than the typical uncertainties and are possibly in a binary system.

Only Stars D and G show a scatter in their radial velocities well above the $2 \sigma$ limit suggested by Nidever et al. (2015) for detecting binary systems. However, the RV scatter of Star II suggests that it too may be in a binary system. If so, the binary nature of this star does not seem to have affected either its optical analysis, nor our analysis of the corrected IR spectra, since the stellar parameters and chemical abundance ratios are in good agreement with other stars in Pal1. It should be noted that the binarity of Star D, E, $\mathrm{F}$ and $\mathrm{H}$ cannot be conclusively established as the sample sizes of these stars are small.

\subsection{DR13}

In the SDSS DR13 release, an attempt to unweight spectra with the persistence problem was established to improve the combined spectral analyses (Albareti et al. 2016). When we examine the DR13 database, two more objects could be added to our analysis; however, the results for Stars I and II are still significantly different from the optical results (see Table 4). We did not pursue the DR13 data release further. The persistence problem is indeed well named.

\section{SUMMARY}

Two members of the unusual star cluster Pal1 have been observed in the APOGEE survey. Examination of their ASPCAP database results are in very poor agreement with previously determined optical analysis. We trace this problem to the known persistence problem that affects up to $30 \%$ of the spectra in the APOGEE database. By removing those spectra with persistence (and other reduction problems), we have re-analysed the cleaned spectra. Our new analyses for the APOGEE spectra of Stars I and II are in excellent agreement with the optical analysis by Sakari et al. (2011). One star, Star F, may be a member of Pal1, based on its heliocentric radial velocity, metallicity and chemical abundances, and location in the tidal tails. However, the temperature of this star is highly uncertain, and it may be (or be contaminated by) an AGB star. All other candidate members found in the APOGEE DR12 database appear to be part of the smooth Galactic background.

\section{ACKNOWLEDGMENTS}

We would like to thank the anonymous referee for the constructive comments and thorough review of this manuscript. F.J. and K.A.V. acknowledge funding for this work through the NSERC Discovery Grants program. C.M.S. acknowledges funding from the Kenilworth Fund of the New York Community Trust. 


\section{REFERENCES}

Albareti F. D., et al., 2016, arXiv preprint arXiv:1608.02013

Belokurov V., et al., 2007, The Astrophysical Journal, 658, 337

Bensby T., Feltzing S., Oey M., 2014, Astronomy \& Astrophysics, 562, A71

Bonifacio P., Sbordone L., Marconi G., Pasquini L., Hill V., 2004, Astronomy \& Astrophysics, 414, 503

Bovy J., Erkal D., Sanders J. L., 2016a, Monthly Notices of the Royal Astronomical Society, p. stw3067

Bovy J., Bahmanyar A., Fritz T. K., Kallivayalil N., 2016b, The Astrophysical Journal, 833, 31

Carlberg R., Grillmair C., Hetherington N., 2012, The Astrophysical Journal, 760, 75

Carretta E., et al., 2010, Astronomy \& Astrophysics, 520, A95

Casagrande L., Ramírez I., Melendez J., Bessell M., Asplund M., 2010, Astronomy \& Astrophysics, 512, A54

Chou M.-Y., Majewski S. R., Cunha K., Smith V. V., Patterson R. J., Martínez-Delgado D., 2010, The Astrophysical Journal Letters, 720, L5

Cohen J. G., 2004, The Astronomical Journal, 127, 1545

Doi M., et al., 2010, The Astronomical Journal, 139, 1628

Dotter A., Chaboyer B., Jevremović D., Kostov V., Baron E., Ferguson J. W., 2008, The Astrophysical Journal Supplement Series, 178,89

Frebel A., Norris J. E., 2015, Annual Review of Astronomy and Astrophysics, 53, 631

Grillmair C., 2006, The Astrophysical Journal Letters, 645, L37

Gunn J. E., et al., 2006, The Astronomical Journal, 131, 2332

Harris W. E., 1996, The Astronomical Journal, 112, 1487

Hawkins K., Masseron T., Jofre P., Gilmore G., Elsworth Y., Hekker S., 2016, Astronomy \& Astrophysics, 594, A43

Hill V., François P., Spite M., Primas F., Spite F., 2000, arXiv preprint astro-ph/0009273

Hinkle K., Wallace L., Livingston W., 2003, in Bulletin of the American Astronomical Society. p. 1260

Holtzman J. A., et al., 2015, The Astronomical Journal, 150, 148

Ishigaki M. N., Hwang N., Chiba M., Aoki W., 2016, The Astrophysical Journal, 823, 157

Jordi K., Grebel E. K., Ammon K., 2006, Astronomy \& Astrophysics, 460, 339

Koesterke L., Prieto C. A., Lambert D. L., 2008, The Astrophysical Journal, 680, 764

Koesterke L., Hubeny I., Stone J. M., MacGregor K., Werner K., 2009, in AIP Conference Proceedings. pp 73-84

Majewski S., Schiavon R., Frinchaboy P., et al. 2015, arXiv preprint arXiv:1701.03802

Martin N., Ibata R., Conn B., Lewis G., Bellazzini M., Irwin M., McConnachie A., 2004, Monthly Notices of the Royal Astronomical Society, 355, L33

Monaco L., Saviane I., Correnti M., Bonifacio P., Geisler D., 2011, Astronomy \& Astrophysics, 525, A124

Mucciarelli A., Carretta E., Origlia L., Ferraro F. R., 2008, The Astronomical Journal, 136, 375

Nidever D. L., et al., 2015, The Astronomical Journal, 150, 173

Niederste-Ostholt M., Belokurov V., Evans N., Koposov S., Gieles M., Irwin M., 2010, Monthly Notices of the Royal Astronomical Society: Letters, 408, L66

Pérez A. E. G., et al., 2016, The Astronomical Journal, 151, 144

Prieto C. A., Beers T. C., Wilhelm R., Newberg H. J., Rockosi C. M., Yanny B., Lee Y. S., 2006, The Astrophysical Journal, 636,804

Ramírez I., Meléndez J., 2005, The Astrophysical Journal, 626, 465

Robin A. C., Reylé C., Derrière S., Picaud S., 2003, Astronomy \& Astrophysics, 409, 523

Sakari C. M., Venn K. A., Irwin M., Aoki W., Arimoto N., Dotter A., 2011, The Astrophysical Journal, 740, 106
Sakari C., McWilliam A., Wallerstein G., 2017, arXiv preprint arXiv:1701.03802

Salaris M., Weiss A., 2002, Astronomy \& Astrophysics, 388, 492

Sarajedini A., et al., 2007, The Astronomical Journal, 133, 1658

Sbordone L., Bonifacio P., Buonanno R., Marconi G., Monaco L., Zaggia S., 2007, Astronomy \& Astrophysics, 465, 815

Schlafly E. F., Finkbeiner D. P., 2011, The Astrophysical Journal, 737,103

Siegel M. H., et al., 2007, The Astrophysical Journal Letters, 667, L57

Tolstoy E., Hill V., Tosi M., 2009, Annual Review of Astronomy and Astrophysics, 47, 371

Venn K. A., Irwin M., Shetrone M. D., Tout C. A., Hill V., Tolstoy E., 2004, The Astronomical Journal, 128, 1177

Venn K. A., et al., 2012, The Astrophysical Journal, 751, 102

Ventura P., D'Antona F., 2008, Astronomy \& Astrophysics, 479, 805

Villanova S., Geisler D., Carraro G., Bidin C. M., Muñoz C., 2013, The Astrophysical Journal, 778, 186

Zasowski G., et al., 2013, The Astronomical Journal, 146, 81

\section{APPENDIX A: SPECTRAL LINES AND ABUNDANCES}

This paper has been typeset from a $\mathrm{T}_{\mathrm{EX}}$ /LATEX file prepared by the author. 
Table A1. Atomic line data and FERRE $[\mathrm{X} / \mathrm{Fe}]^{a}$ ratios

\begin{tabular}{|c|c|c|c|c|c|c|c|c|c|}
\hline Element & Lambda $(\AA)$ & Star I & Star II & Star D & Star E & Star F & Star F* & Star G & Star H \\
\hline \multirow[t]{56}{*}{ Fe I } & 15211.682 & -1.0 & -0.6 & - & -0.7 & -1.0 & $<-1.7$ & -0.6 & -0.4 \\
\hline & 15249.140 & -0.6 & -0.6 & -1.0 & -0.7 & -0.6 & -1.3 & -0.4 & -0.4 \\
\hline & 15297.317 & -0.4 & $>-0.4$ & $>-1.0$ & -0.7 & - & - & - & -0.2 \\
\hline & 15309.789 & - & - & - & $\sim-0.8$ & -0.4 & - & - & -0.2 \\
\hline & 15339.574 & -0.8 & -0.6 & -0.9 & -0.6 & -1.0 & $<-1.8$ & -0.4 & -0.2 \\
\hline & 15392.011 & -0.8 & -0.6 & -0.8 & -0.6 & -0.6 & $<-1.8$ & -0.8 & -0.6 \\
\hline & 15483.107 & -0.6 & -0.4 & - & -0.6 & - & - & - & -0.2 \\
\hline & 15494.762 & -0.6 & -0.6 & -0.6 & -0.6 & -0.6 & - & - & -0.4 \\
\hline & 15505.316 & - & - & -0.6 & -0.6 & -0.6 & -1.4 & -0.6 & -0.4 \\
\hline & 15528.553 & - & - & -0.4 & -0.7 & - & - & - & - \\
\hline & 15546.326 & - & - & $>-0.9$ & -0.5 & -0.6 & -1.4 & -0.4 & - \\
\hline & 15595.760 & -0.8 & -0.6 & -1.0 & -0.7 & $<-0.8$ & -1.6 & - & -0.3 \\
\hline & 15608.487 & -0.8 & -0.8 & $>-1.0$ & -0.8 & -0.6 & -1.6 & -0.6 & -0.4 \\
\hline & 15615.412 & - & -0.6 & - & - & - & $<-1.8$ & -0.6 & -0.6 \\
\hline & 15666.296 & -0.8 & -0.8 & - & -0.7 & $<-0.7$ & $<-1.8$ & -0.5 & -0.6 \\
\hline & 15681.805 & -0.7 & -0.4 & -1.0 & -0.6 & -0.6 & - & -0.5 & -0.4 \\
\hline & 15735.713 & - & - & - & - & - & - & - & - \\
\hline & 15765.622 & - & - & - & -0.4 & -0.6 & - & - & -0.2 \\
\hline & 15778.381 & -0.4 & -0.6 & -1.0 & -0.5 & -0.6 & -1.2 & - & -0.4 \\
\hline & 15899.571 & -0.6 & - & -0.4 & $>-0.4$ & - & - & -0.5 & -0.4 \\
\hline & 15905.797 & -0.6 & - & -0.4 & -0.4 & - & - & - & -0.4 \\
\hline & 15910.390 & -0.6 & -0.4 & -0.4 & -0.5 & -0.8 & - & - & -0.4 \\
\hline & 15924.987 & -0.7 & -0.5 & -0.4 & -0.7 & -0.6 & -1.3 & - & -0.4 \\
\hline & 15946.207 & - & - & -0.4 & -0.8 & -0.6 & -1.6 & - & -0.5 \\
\hline & 15958.447 & $<-0.8$ & -0.6 & -0.4 & -0.6 & - & $<-1.4$ & -0.7 & -0.2 \\
\hline & 15969.209 & -0.8 & -0.8 & -0.6 & $<-0.8$ & - & - & -0.8 & -0.6 \\
\hline & 15975.615 & - & - & - & - & -0.4 & - & - & - \\
\hline & 16011.133 & -0.6 & -0.6 & -0.4 & -0.8 & - & -1.4 & $>-0.4$ & -0.6 \\
\hline & 16013.985 & -0.6 & -0.6 & -0.4 & -0.8 & - & - & $>-0.4$ & -0.6 \\
\hline & 16080.311 & -0.5 & -0.6 & - & -0.6 & -0.6 & $<-1.8$ & - & -0.6 \\
\hline & 16130.274 & -0.8 & -0.6 & - & -0.6 & -0.8 & -1.4 & - & -0.4 \\
\hline & 16157.660 & -0.6 & -0.4 & -0.4 & -0.6 & -0.6 & -1.4 & -0.4 & -0.4 \\
\hline & 16169.448 & -0.6 & -0.5 & -0.8 & -0.7 & -0.8 & -1.6 & $>-0.4$ & -0.2 \\
\hline & 16190.224 & -0.6 & -0.4 & -1.0 & -0.8 & -0.6 & - & - & -0.6 \\
\hline & 16212.175 & -0.7 & -0.4 & -0.8 & -0.6 & -0.8 & - & -0.8 & -0.4 \\
\hline & 16217.970 & -0.4 & -0.4 & -0.8 & -0.7 & - & -1.2 & -0.4 & -0.4 \\
\hline & 16236.084 & -0.8 & -0.6 & - & -0.7 & -0.8 & $<-1.8$ & - & -0.6 \\
\hline & 16240.487 & - & - & - & - & - & $<-1.8$ & -0.4 & - \\
\hline & 16256.993 & - & - & -0.4 & $<-0.8$ & -0.6 & - & - & - \\
\hline & 16297.294 & -0.6 & - & - & -0.8 & -0.6 & -1.4 & -0.4 & -0.6 \\
\hline & 16320.829 & - & - & -0.8 & -0.6 & -0.8 & -1.5 & - & -0.6 \\
\hline & 16328.912 & -0.7 & -0.4 & -0.4 & -0.8 & -0.8 & $<-1.4$ & - & -0.6 \\
\hline & 16402.650 & -0.6 & -0.6 & -0.8 & -0.8 & - & $<-1.8$ & -0.6 & -0.6 \\
\hline & 16409.869 & - & - & - & - & - & - & - & - \\
\hline & 16510.805 & -0.6 & -0.4 & -0.8 & -0.6 & - & -1.4 & -0.4 & -0.6 \\
\hline & 16521.738 & -0.5 & -0.6 & -0.4 & -0.6 & -0.4 & $>1.4$ & $>-0.4$ & -0.2 \\
\hline & 16536.502 & -0.6 & - & -0.6 & -0.6 & -0.4 & -1.4 & -0.6 & -0.4 \\
\hline & 16556.519 & -0.6 & -0.5 & -0.4 & -0.6 & -0.3 & - & - & -0.4 \\
\hline & 16590.582 & - & - & - & - & - & - & - & - \\
\hline & 16617.302 & - & - & $>-1.0$ & $<-0.8$ & - & $<-1.8$ & -0.6 & $<-0.6$ \\
\hline & 16624.278 & - & - & -1.0 & -0.7 & - & - & - & -0.6 \\
\hline & 16650.424 & -0.4 & -0.4 & -0.6 & -0.6 & -0.3 & -1.4 & $>-0.4$ & -0.4 \\
\hline & 16670.037 & $>-0.4$ & $>-0.4$ & -0.8 & -0.3 & -0.3 & -1.4 & - & -0.4 \\
\hline & 16757.644 & - & -0.8 & -0.4 & -0.6 & -0.3 & - & - & -0.2 \\
\hline & 16804.240 & - & - & -0.4 & -0.6 & - & - & - & -0.4 \\
\hline & 16848.118 & - & - & -0.8 & $<-0.8$ & - & $<-1.8$ & - & - \\
\hline \multirow[t]{5}{*}{$\mathrm{O}$ from $\mathrm{OH}$ lines } & 15241.164 & - & - & - & 0.0 & - & - & - & 0.2 \\
\hline & 15396.206 & - & - & - & - & -0.2 & - & - & 0.4 \\
\hline & 15413.211 & 0.4 & 0.2 & 0.5 & $>0.5$ & $<-0.2$ & - & - & 0.2 \\
\hline & 15509.737 & - & - & 0.2 & $>0.5$ & - & $<-0.2$ & 0.1 & 0.3 \\
\hline & 15564.252 & - & 0.2 & 0.1 & 0.3 & -0.4 & - & 0.2 & $<-0.2$ \\
\hline
\end{tabular}


Table A1 - continued

\begin{tabular}{|c|c|c|c|c|c|c|c|c|c|}
\hline Element & Lambda $(\AA)$ & Star I & Star II & Star D & Star E & Star F & Star F* & Star G & Star H \\
\hline & 15573.254 & - & - & 0.2 & 0.7 & - & - & - & 0.4 \\
\hline & 15576.255 & - & - & - & 0.7 & $<0.0$ & - & - & - \\
\hline & 15631.270 & - & - & - & 0.7 & - & - & - & - \\
\hline & 16056.386 & 0.5 & - & - & 0.7 & - & - & - & -0.2 \\
\hline & 16065.388 & 0.5 & 0.2 & 0.2 & -0.2 & -0.3 & 0.0 & 0.2 & -0.2 \\
\hline & 16069.389 & - & - & - & - & - & - & - & $0.4<$ \\
\hline & 16196.424 & - & - & - & - & - & - & - & - \\
\hline & 16256.440 & - & - & - & - & - & - & 0.0 & - \\
\hline & 16264.442 & - & - & - & - & - & - & - & - \\
\hline & 16350.466 & 0.5 & 0.2 & 0.5 & 0.3 & 0.2 & - & - & 0.2 \\
\hline & 16356.467 & - & - & - & - & - & - & - & - \\
\hline & 16358.467 & - & 0.5 & 0.4 & 0.3 & $<0.2$ & - & - & - \\
\hline & 16372.472 & $>0.6$ & - & 0.2 & 0.7 & - & $<-0.2$ & - & - \\
\hline & 16530.515 & - & - & - & - & - & $<-0.2$ & - & - \\
\hline & 16539.517 & $>0.6$ & - & $>0.2$ & 0.7 & - & - & - & 0.2 \\
\hline & 16543.518 & - & - & - & - & - & 0.0 & 0.2 & - \\
\hline & 16708.563 & 0.5 & - & 0.2 & 0.7 & - & $<-0.2$ & - & 0.4 \\
\hline & 16718.566 & $>0.5$ & 0.5 & 0.5 & 0.7 & - & $<-0.2$ & - & 0.4 \\
\hline \multirow[t]{6}{*}{$\mathrm{C}$ from $\mathrm{CO}$ lines } & 15325.187 & - & $<0.1$ & 0.1 & - & - & - & - & 0.2 \\
\hline & 15367.243 & - & - & $<0.2$ & 0.1 & 0.2 & - & $<0.2$ & -0.1 \\
\hline & 15470.226 & - & - & - & - & 0.4 & - & - & 0.2 \\
\hline & 15499.690 & - & $<0.0$ & - & - & - & - & - & 0.2 \\
\hline & 16186.421 & $<0.0$ & $<0.2$ & 0.1 & 0.0 & - & - & - & - \\
\hline & 16193.923 & - & - & - & - & 0.2 & - & - & 0.0 \\
\hline \multirow[t]{4}{*}{ MgI } & 15745.017 & 0.0 & 0.0 & -0.2 & 0.1 & -0.2 & -0.2 & 0.8 & 0.5 \\
\hline & 15753.189 & -0.2 & -0.1 & -0.1 & 0.3 & -0.5 & -0.6 & 0.8 & 0.5 \\
\hline & 15770.150 & -0.3 & -0.2 & -0.4 & 0.3 & - & -0.6 & 0.7 & 0.5 \\
\hline & 15958.836 & - & 0.1 & 0.3 & 0.2 & -0.2 & - & $<0.4$ & 0.3 \\
\hline \multirow[t]{3}{*}{$\mathrm{MnI}$} & 15222.009 & -0.1 & $<0.0$ & -0.2 & 0.1 & 0.4 & 0.3 & 0.2 & 0.2 \\
\hline & 15791.657 & - & - & 0.0 & 0.0 & - & $<0.0$ & - & - \\
\hline & 15969.543 & - & -0.1 & -0.2 & -0.2 & 0.1 & 0.2 & -0.3 & -0.1 \\
\hline \multirow[t]{3}{*}{$\mathrm{CaI}$} & 16161.778 & 0.2 & 0.4 & 0.3 & 0.2 & -0.2 & - & - & 0.1 \\
\hline & 16208.514 & $<0.0$ & 0.0 & 0.4 & 0.3 & -0.4 & - & - & - \\
\hline & 16213.018 & - & - & 0.0 & 0.0 & - & - & - & - \\
\hline \multirow[t]{3}{*}{ SI } & 15426.404 & 0.2 & $<0.1$ & 0.0 & 0.6 & - & - & - & 0.2 \\
\hline & 15474.047 & - & -0.1 & - & 0.0 & -0.3 & -0.2 & - & 0.1 \\
\hline & 15482.710 & 0.1 & 0.2 & 0.2 & -0.2 & -0.5 & - & - & 0.3 \\
\hline \multirow[t]{2}{*}{ AlI } & 16723.527 & -0.1 & -0.1 & 0.4 & 0.3 & 0.8 & 0.8 & $<0.7$ & 0.3 \\
\hline & 16767.939 & 0.2 & $<0.2$ & 0.4 & 0.3 & 0.7 & 0.8 & - & 0.3 \\
\hline KI & 15172.521 & 0.1 & 0.2 & -0.2 & 0.0 & 0.1 & -0.2 & 0.7 & $>0.0$ \\
\hline \multirow[t]{2}{*}{$\mathrm{NaI}$} & 16378.326 & - & - & - & $>0.1$ & - & - & - & - \\
\hline & 16393.327 & - & - & - & - & $>-0.4$ & $<-0.4$ & - & - \\
\hline \multirow[t]{9}{*}{ SiI } & 15562.031 & $<-0.2$ & 0.2 & - & 0.2 & - & - & 0.2 & - \\
\hline & 16055.585 & - & - & - & - & - & - & - & - \\
\hline & 16064.397 & -0.2 & 0.0 & 0.0 & -0.2 & - & - & - & 0.0 \\
\hline & 16099.184 & $<-0.2$ & 0.2 & 0.4 & 0.0 & -0.4 & -0.4 & $<0$ & 0.2 \\
\hline & 16185.583 & -0.2 & - & 0.0 & 0.2 & - & - & 0.0 & 0.1 \\
\hline & 16191.217 & - & - & - & - & - & - & - & - \\
\hline & 16220.100 & 0.0 & 0.2 & -0.1 & -0.2 & -0.4 & -0.4 & 0.0 & 0.2 \\
\hline & 16685.327 & 0.2 & 0.2 & 0.2 & 0.0 & 0.4 & 0.4 & $0.2<$ & 0.2 \\
\hline & 16832.756 & 0.0 & - & 0.2 & 0.0 & 0.4 & 0.4 & 0.2 & 0.0 \\
\hline
\end{tabular}

${ }^{a}[\mathrm{X} / \mathrm{H}]$ is given instead for Fe I. 